This is an electronic reprint of the original article. This reprint may differ from the original in pagination and typographic detail.

Author(s): Banichuk, Nikolay; Jeronen, Juha; Neittaanmäki, Pekka; Tuovinen, Tero

Title: $\quad$ Dynamic Behaviour of an Axially Moving Plate Undergoing Small Cylindrical Deformation Submerged in Axially Flowing Ideal Fluid

Year: $\quad 2011$

Version:

Please cite the original version:

Banichuk, N., Jeronen, J., Neittaanmäki, P., \& Tuovinen, T. (2011). Dynamic Behaviour of an Axially Moving Plate Undergoing Small Cylindrical Deformation Submerged in Axially Flowing Ideal Fluid. Journal of Fluids and Structures, 27(7), 986-1005. https://doi.org/10.1016/j.jfluidstructs.2011.07.004

All material supplied via JYX is protected by copyright and other intellectual property rights, and duplication or sale of all or part of any of the repository collections is not permitted, except that material may be duplicated by you for your research use or educational purposes in electronic or print form. You must obtain permission for any other use. Electronic or print copies may not be offered, whether for sale or otherwise to anyone who is not an authorised user. 


\title{
Dynamic behaviour of an axially moving plate undergoing small cylindrical deformation submerged in axially flowing ideal fluid
}

\author{
N. Banichuk ${ }^{\mathrm{a}}$, J. Jeronen ${ }^{\mathrm{b}, *}$, \\ P. Neittaanmäki ${ }^{b}$ and T. Tuovinen ${ }^{\mathrm{b}}$ \\ a Institute for Problems in Mechanics RAS, prosp. Vernadskogo 101, block 1, Moscow \\ 119526, Russian Federation \\ ${ }^{\mathrm{b}}$ Department of Mathematical Information Technology, Mattilanniemi 2 (Agora), 40014 \\ University of Jyväskylä, Finland
}

\begin{abstract}
The out-of-plane dynamic response of a moving plate, travelling between two rollers at a constant velocity, is studied, taking into account the mutual interaction between the vibrating plate and the surrounding, axially flowing ideal fluid. Transverse displacement of the plate (assumed cylindrical), is described by an integrodifferential equation that includes a local inertia term, Coriolis and centrifugal forces, the aerodynamic reaction of the external medium, the vertical projection of membrane tension, the bending resistance, and external perturbation forces. In the two-dimensional model thus set up, the aerodynamic reaction is found analytically as a functional of the cylindrical displacement, using the techniques of complex analysis. The resulting integro-differential problem is discretized in space with the Fourier-Galerkin method, and integrated in time with the diagonalization method. Examples are computed with physical parameters corresponding to air and some paper materials. The effects of the surrounding fluid on the critical velocity and first natural frequency are investigated, for stationary air, for an air mass moving with the plate, and for some arbitrary axial fluid velocities. The obtained results are applicable for both an ideal membrane and a plate with nonzero bending rigidity.
\end{abstract}

Key words: ideal fluid, axially moving plate, flat panel, dynamics, FSI, paper web

\footnotetext{
* Corresponding author. Tel.: +358 40760 7328; fax: +358 142602771.

Email address: juha. jeronen@jyu.fi (J. Jeronen).
} 


\section{Introduction}

An extensive amount of research has been conducted on travelling flexible strings, membranes, beams and plates, which are the most common models of axially moving continua. See e.g. Archibald and Emslie (1958), Miranker (1960), Swope and Ames (1963), Mote (1968, 1972, 1975), Simpson (1973), Ulsoy and Mote (1980, 1982), Chonan (1986), Wickert and Mote (1990), Lin and Mote (1995), Shen et al. (1995), Wang (2003), Shin et al. (2005), Sygulski (2007), and Kulachenko et al. (2007a,b).

These studies used a model described by second- and fourth-order differential equations, and focused on various aspects of free vibrations and forced vibrations. Stability considerations were reviewed in Mote (1972). The effects of axial motion on the frequency spectrum and eigenfunctions were investigated in Archibald and Emslie (1958) and Simpson (1973). It was shown that the natural frequency of each mode decreases when transport speed increases, and that the travelling string and beam both experience divergence instability at a sufficiently high speed. Response prediction has been given for particular cases when excitation assumes special forms, such as a constant transverse point force (Chonan, 1986) or harmonic support motion (Miranker, 1960). Arbitrary excitation and initial conditions have been analysed with the help of modal analysis and a Green function method in Wickert and Mote (1990). As a result, the associated critical speeds have been determined explicitly.

In most contexts, the standard approach to dynamic fluid-structure interaction problems during the last decade has been numerical simulation with the Navier-Stokes equations numerically coupled with an elasticity model. The elasticity model may be nonlinear and allow large deformations. For example, Stein et al. $(2000,2001)$ and Sathe et al. (2007) have studied complex fluid-structure interaction in parachutes. In the context of paper production however, both two- and three-dimensional approaches, as also various different approaches to taking into account the effect of the surrounding fluid, have been used. As we can see from Chang and Moretti (1991) and Watanabe et al. (2002), potential flow analyses play an important part in investigation of dynamic fluid-structure interaction in paper production. Because the paper web is fragile, linear theory is sufficient to cover the physically meaningful range of deformations up to the first instability.

The first attempt to take into account the interaction between a travelling paper web and the external medium, using the finite element method, was made in the series of papers by Niemi and Pramila (1986) and Pramila $(1986,1987)$. It was found that air significantly reduces the eigenfrequencies and critical velocities of the web when compared to the vacuum case. In Chang and Moretti (1991), the web was modelled as a linear membrane, while the surrounding air was treated using potential flow theory. A nonlin- 
ear membrane element was used in Koivurova and Pramila (1997) to model a narrow axially moving band surrounded by air. The influence of air was accounted for with acoustic elements placed on one side of the band only. In Kulachenko et al. (2007a), the influence of air was accounted for by utilising fluid-solid interaction analysis based on acoustic theory. Results in this paper also show that air reduces the eigenfrequencies of the web.

In Watanabe et al. (2002), two different methods of analysis were developed in order to clarify the phenomenon of paper flutter. One of these was a flutter simulation using a Navier-Stokes code, and the other method was based on a potential flow analysis of an oscillating thin airfoil. In $\mathrm{Wu}$ and Kaneko (2005), both linear and nonlinear analyses of sheet flutter caused by fluidstructure interaction in a narrow passage were developed. The sheet was considered as a combination of massless beam elements, springs, and discrete mass particles, in which the mass of each particle and the spring coefficients were calculated based on the beam model.

In a recent paper, Frondelius et al. (2006) contrasted the results of Pramila (1986) with a model including the boundary layer. They reported that while the eigenfrequency predictions agree with those from potential flow theory, the divergence velocities are found to be significantly higher. Nevertheless, from an academic research viewpoint, the potential flow problem remains a standard reference case. It has been studied for axially moving materials in stationary air (e.g., Pramila 1986), and for stationary structures in axial flow (e.g., Eloy et al. 2007, Howell et al. 2011, Doaré et al. 2011).

It should be noted that the form of the present problem shares some similarities with the problems of pipes conveying fluid, and stationary structures subjected to axial flow. These similarities between the two mentioned areas have been discussed in the review by Païdoussis (2008). A functional analytical solution for the reaction of the fluid has been found for a stationary plate in axial flow by Kornecki et al. (1976). See also Dugundji et al. (1963), Guo and Païdoussis (2000), and Firouz-Abadi et al. (2010), and for a summary comparing the different results, the book by Païdoussis (2004).

In the present paper, we extend into the dynamic case our earlier study, Banichuk et al. (2010b), where the static instability of a travelling plate, undergoing small cylindrical deformation (see, e.g., Timoshenko and Woinowsky-Krieger 1959) and subjected to axial flow of ideal fluid, was studied. In the present study, we use an approach similar to Kornecki et al. (1976), deriving the aerodynamic reaction analytically as a functional of the displacement, for the case where both the plate and the fluid are allowed to move axially, and flow occurs on both sides of the plate. We then proceed with a numerical solution of the resulting integro-differential equation. Our numerical approach is based on a Fourier-Galerkin discretization in space, and a diagonalization method (see, e.g., Kreyszig 1993) in time. 
The Fourier-Galerkin method is a traditional spectral Galerkin method. According to Canuto et al. (1988), the first serious application of spectral methods to partial differential equations was made by Silberman (1954) for meteorological modelling. For a stability analysis of the method and some further references, see Tadmor (1987). Recently, the method has been applied, e.g., in computing the stationary solutions of two-dimensional generalised wave equations (Christou and Christov, 2007).

The Fourier-Galerkin method is well suited for problems with periodic boundary conditions and simple geometry (Canuto et al., 1988). While our problem is not periodic in the strict sense, the method is applicable because we have the same boundary conditions on both ends of the domain, and thus $w(-\ell)=w(\ell)$.

Note that our solution for the aerodynamic problem does not require these exact boundary conditions. Any choice of boundary conditions can be used, as long as the displacement $w$ and its derivatives inside the domain are small. For one example, the same aerodynamic solution is applicable also with the nonsymmetric boundary conditions introduced by Garziera and Amabili (2000), where the case of tape winding onto a reel was investigated.

Finally, it should be noted that in the particular context of paper production, the present study ignores some potentially important effects. First of all, the cylindrical deformation assumption is a reasonable approximation only for narrow strips, as was noted in our previous study, Banichuk et al. (2010b). This is explained by the form of the problem: even for the static case in a vacuum, it can be shown (for details, see our earlier study, Banichuk et al. 2010a) that for a pinned-free plate, the deformation becomes localised in the vicinity of the free boundaries, and that the buckling shape is that of eigenfunctions of free vibrations of a stationary rectangular plate (given, e.g., in Gorman 1982). Secondly, the viscoelasticity, which introduces damping into the system, is ignored. Thus, the real system is approximated with a conservative one. This is not expected to change the critical velocity, but it does modify the postdivergence behaviour (Ulsoy and Mote, 1982), and the dynamic response. Note, however, that if the boundary conditions are not symmetric, dissipation may have either a stabilizing or an instabilizing effect. See e.g. Païdoussis (1998), Sugiyama and Langthjem (2007), and Doaré (2010).

\section{Dynamic problem of a travelling plate submerged in ideal fluid}

Consider a travelling paper web interacting with air (Figure 1). The dynamics of the web is investigated with the help of the model of an elastic plate, moving in the $x$ direction with constant velocity $V_{0}$. The surrounding 


\section{Insert Figure 1 here}

Figure 1. Travelling plate undergoing cylindrical deformation, supported at $x=-\ell$ and $x=+\ell$, and interacting with air. At infinity, the air moves along the $x$ axis with velocity $v_{\infty}$.

medium is modelled as an ideal fluid. Thus the effects of viscosity, compression, and fluid rotation are neglected. An orthogonal xyz coordinate system is used, and all investigated behavioural values describing the process of the plate vibrations are assumed to be functions independent of the coordinate $y$.

In other words, we consider a plane problem of aero-elastic dynamic interaction of the fluid and the plate. That is, the plate is described by the flat panel model (Bisplinghoff and Ashley, 1962). Note that although the flat panel model has a different physical interpretation than the beam model, they share the mathematical formulation (with bending rigidity $D$ replacing the beam rigidity $E I$ ).

The function $w=w(x, t)$, describing small transverse cylindrical displacements of the plate, and the aerodynamic forces $q_{f}(x, t)$ applied to the plate, are related by the equation of transverse vibrations (see, e.g., Mote 1972, Chang and Moretti 1991, Kurki et al. 1995)

$$
m \frac{\partial^{2} w}{\partial t^{2}}+2 m V_{0} \frac{\partial^{2} w}{\partial x \partial t}+\left(m V_{0}^{2}-T\right) \frac{\partial^{2} w}{\partial x^{2}}+D \frac{\partial^{4} w}{\partial x^{4}}=q_{f}+g,
$$

where $m$ is the mass of the plate per unit area, $V_{0}$ the axial velocity, $T$ the applied tension, and $D$ the bending rigidity (Timoshenko and Woinowsky-Krieger, 1959)

$$
D=\frac{E h^{3}}{12\left(1-v^{2}\right)},
$$

where $E$ is the Young's modulus, $h$ the thickness of the plate, and $v$ the Poisson ratio. Finally, the function $g=g(x, t)$ in (1) is a given external disturbance.

The problem is considered with the simply supported (pinned) boundary conditions

$$
w(-\ell, t)=\frac{\partial^{2} w}{\partial x^{2}}(-\ell, t)=0, w(\ell, t)=\frac{\partial^{2} w}{\partial x^{2}}(\ell, t)=0,
$$

and the initial conditions

$$
w(x, 0)=g_{1}(x), \frac{\partial w}{\partial t}(x, 0)=g_{2}(x), \quad x \in[-\ell, \ell] .
$$

The functions $g_{1}=g_{1}(x), g_{2}=g_{2}(x)$ are, respectively, the given initial displacement and initial transverse velocity. In the case of an ideal membrane $(D=0)$, the curvature boundary conditions are not needed. 
We will consider the problem in the dimensionless coordinates

$$
\begin{aligned}
x^{\prime} & \equiv x / \ell, \quad x^{\prime} \in[-1,1] \\
t^{\prime} & \equiv t / \tau, \quad t^{\prime} \in[0, \infty)
\end{aligned}
$$

where $\ell$ is the half-length of the span (see Figure 1) and $\tau$ is an arbitrary timescale parameter. Additionally, let us define

$$
w^{\prime}\left(x^{\prime}, t^{\prime}\right) \equiv w(x, t)
$$

and in the same manner for $q_{f}(x, t)$ and $g(x, t)$. In the following, the primes will be omitted, and unless otherwise stated, all coordinates refer to the dimensionless forms (5)-(6).

We consider nonstationary aerodynamic flow in two dimensions. As our coordinate system, we choose the $x z$ plane with Cartesian coordinates, setting the $x$ axis parallel to the flow of the fluid and the movement of the plate.

The aerodynamic velocity potential $\Phi(x, z, t)$ of airflow with respect to the moving plate surface and total pressure $P(x, z, t)$ have the forms

$$
\Phi(x, z, t)=x v_{\infty}+\varphi(x, z, t)
$$

and

$$
P(x, z, t)=p_{\infty}+p(x, z, t) .
$$

Here $v_{\infty}$ and $p_{\infty}$ are, respectively, the given velocity and pressure at infinity, and $\varphi$ and $p$ are aerodynamic disturbances of the velocity potential and pressure.

Total reaction force $q_{f}$ exerted by the fluid is equal to the difference of pressure between the upper and lower faces of the plate,

$$
q_{f}(x, t)=P^{-}(x, t)-P^{+}(x, t)=p^{-}(x, t)-p^{+}(x, t), \quad-1 \leq x \leq 1 .
$$

The \pm superscript notation used here is defined as

$$
f^{ \pm}(x, t) \equiv \lim _{z \rightarrow 0^{ \pm}} f(x, z, t) ，
$$

where $f$ is any function and the upper (lower) signs correspond to each other.

Regarding $\varphi$ and $w$ and their first derivatives as small, the linearised aerodynamic reaction can be represented as

$$
q_{f}=p^{-}-p^{+}=\rho_{f}\left(\frac{\partial}{\partial t}+v_{\infty} \frac{\partial}{\partial x}\right)\left(\varphi^{+}(x, t)-\varphi^{-}(x, t)\right) .
$$


In order to find out the final expression for $q_{f}$, we need to solve the aerodynamic disturbance potential $\varphi$.

We specify the boundary condition that the fluid does not cross the plate surface. Taking into account that for the components of the unit normal vector, $n_{x} \approx-\partial w / \partial x$ and $n_{z} \approx 1$, we obtain approximate linear expressions for the normal components of the fluid and plate velocities:

$$
v_{n}=v_{\infty} n_{x}+\frac{\partial \varphi}{\partial z} n_{z}, \quad\left(\frac{\mathrm{d} \mathbf{U}}{\mathrm{d} t}\right)_{n}=\frac{\partial w}{\partial t},
$$

where, in the first expression, we have omitted the second-order small term $n_{x}(\partial \varphi / \partial x)=-(\partial \varphi / \partial x)(\partial w / \partial x)$. Using (12), we have the following linearised expression for this boundary condition:

$$
\left.\frac{\partial \varphi}{\partial z}\right|_{z=0,-1 \leq x \leq 1}=\frac{\partial w}{\partial t}+v_{\infty} \frac{\partial w}{\partial x} \equiv \gamma(x, t) .
$$

The linearised aerodynamic problem can then be written as

$$
\begin{aligned}
\triangle \varphi & \equiv \frac{\partial^{2} \varphi}{\partial x^{2}}+\frac{\partial^{2} \varphi}{\partial z^{2}}=0 \\
\left(\frac{\partial \varphi}{\partial z}\right)^{ \pm} & =\gamma(x, t), \quad z=0,-1 \leq x \leq 1 \\
(\nabla \varphi)_{\infty} & =0,
\end{aligned}
$$

where $\gamma(x, t)$ is defined in (13).

Note that the domain of the aerodynamic problem is infinite. It consists of the whole $x z$ plane with the exception of the cut at $z=0,-1 \leq$ $x \leq 1$, which is our linearised representation of the space occupied by the plate. Although we consider an axially moving plate, for the purposes of our analysis the plate only exists on the interval $-1 \leq x \leq 1$. The solution of this problem, together with equation (11), provides the final expression for $q_{f}$.

\section{Solution of the aerodynamic problem}

In this section, we will solve the aerodynamic problem (14)-(16) analytically on the plate surface, as a functional of the displacement $w$. We will apply the techniques of complex analysis.

We introduce an auxiliary function

$$
W(\eta, t)=\Psi(x, z, t)+\mathrm{i} \varphi(x, z, t)
$$


of the complex variable $\eta=x+\mathrm{i} z$, where $\mathrm{i}^{2}=-1$. The Cauchy-Riemann equations and boundary conditions (15) imply that

$$
\left.\frac{\partial \Psi}{\partial x}\right|_{z=0}=\left.\frac{\partial \varphi}{\partial z}\right|_{z=0}=\gamma(x, t)
$$

and, consequently, we have

$$
\begin{aligned}
& \Psi(x, t)=\chi(x, t)+C(t) \\
& \chi(x, t)=\int_{-1}^{x} \gamma(\xi, t) d \xi,
\end{aligned}
$$

where $C(t)$ is a real constant of integration for each fixed $t$.

Thus, finding the potential reduces to the computation of the imaginary part of the analytic function (17), whose real part on $[-1,1]$ is (19). We use the results given by Sherman (1952) (see also Ashley and Landahl, 1985, chap. 5-3) and represent the solution of this problem as

$$
W(\eta, t)=\frac{1}{2 \pi \mathrm{i}}\left(\frac{\eta-1}{\eta+1}\right)^{1 / 2} \int_{-1}^{1}\left(\frac{\xi+1}{\xi-1}\right)^{1 / 2} \frac{\chi(\xi, t)+C(t)}{\xi-\eta} \mathrm{d} \xi
$$

The real constant $C(t)$ is determined with the help of the following equation:

$$
\frac{1}{2 \pi \mathrm{i}} \int_{-1}^{1} \frac{\chi(\xi, t)+C(t)}{\sqrt{\xi^{2}-1}} \mathrm{~d} \xi=0,
$$

which represents a regularity condition for the function $W$ at the point $\eta=$ 1. From condition (22), we have

$$
C(t)=\frac{1}{\pi \mathrm{i}} \int_{-1}^{1} \frac{\chi(\xi, t) \mathrm{d} \xi}{\sqrt{\xi^{2}-1}}
$$

Using expression (23) and the formula

$$
\frac{1}{2 \pi \mathrm{i}} \int_{-1}^{1}\left(\frac{\xi+1}{\xi-1}\right)^{1 / 2} \frac{\mathrm{d} \xi}{\xi-\eta}=\frac{1}{2}\left(\frac{\eta+1}{\eta-1}\right)^{1 / 2}-\frac{1}{2}
$$

we perform substitutions into expression (21) and elementary transforma- 
tions and obtain

$$
\begin{aligned}
W=\frac{1}{2 \pi \mathrm{i}}\left(\frac{\eta-1}{\eta+1}\right)^{1 / 2} \int_{-1}^{1}\left(\frac{\xi+1}{\xi-1}\right)^{1 / 2} \frac{\chi(\xi, t) \mathrm{d} \xi}{\xi-\eta} \\
\quad+\frac{C(t)}{2}\left[1-\left(\frac{\eta-1}{\eta+1}\right)^{1 / 2}\right] \\
=\frac{\sqrt{\eta^{2}-1}}{2 \pi \mathrm{i}} \int_{-1}^{1} \frac{\chi(\xi, t) \mathrm{d} \xi}{(\xi-\eta) \sqrt{\xi^{2}-1}}+\frac{C(t)}{2} .
\end{aligned}
$$

From the representation (25), we can compute the quantity $\varphi^{+}$:

$$
\begin{aligned}
\varphi^{+} & =\lim _{z \rightarrow 0^{+}}[\operatorname{Im} W(x+\mathrm{i} z)] \\
& =\quad \text { p.v. }\left(-\frac{\sqrt{1-x^{2}}}{2 \pi} \int_{-1}^{1} \frac{\chi(\xi, t) \mathrm{d} \xi}{(\xi-x) \sqrt{1-\xi^{2}}}\right) .
\end{aligned}
$$

Here, we took into account that the constant $C(t)$ on the right-hand side of (25) is real, and consequently must be omitted when the limit of the imaginary part is computed in (26). Note also that the integration in (26) is understood in the sense of Cauchy's principal value (p.v.).

We have

$$
\varphi^{+}-\varphi^{-}=2 \varphi^{+}
$$

because the flow is antisymmetric with respect to the linearised plate surface (see, e.g., Eloy et al. 2007 for a similar case). Alternatively, we can take the corresponding limit of (25) at $\eta=x-\mathrm{i} z \rightarrow x-\mathrm{i} \cdot 0\left(z \rightarrow 0^{-}\right)$and obtain the same result.

By definition of the principal value, we have

$$
\begin{aligned}
2 \varphi^{+}= & \text {p.v. }\left(-\frac{1}{\pi} \int_{-1}^{1}\left(\frac{1-x^{2}}{1-\xi^{2}}\right)^{1 / 2} \frac{\chi(\xi, t) \mathrm{d} \xi}{\xi-x}\right) \\
=\lim _{\varepsilon \rightarrow 0}- & \frac{1}{\pi}\left[\int_{-1}^{x-\varepsilon}\left(\frac{1-x^{2}}{1-\xi^{2}}\right)^{1 / 2} \frac{\chi(\xi, t) \mathrm{d} \xi}{\xi-x}\right. \\
& \left.+\int_{x+\varepsilon}^{1}\left(\frac{1-x^{2}}{1-\xi^{2}}\right)^{1 / 2} \frac{\chi(\xi, t) \mathrm{d} \xi}{\xi-x}\right] .
\end{aligned}
$$

Integrating by parts, and substituting expression (20) for $\chi(x)$, we 
have

$$
\begin{aligned}
2 \varphi^{+}=\lim _{\varepsilon \rightarrow 0}[ & N(x-\varepsilon, x) \int_{-1}^{x-\varepsilon} \gamma(\xi, t) \mathrm{d} \xi \\
& -N(x+\varepsilon, x) \int_{-1}^{x+\varepsilon} \gamma(\xi, t) \mathrm{d} \xi \\
& -\int_{-1}^{x-\varepsilon} N(\xi, x) \gamma(\xi, t) \mathrm{d} \xi \\
& \left.-\int_{x+\varepsilon}^{1} N(\xi, x) \gamma(\xi, t) \mathrm{d} \xi\right],
\end{aligned}
$$

where we use the notation

$$
\begin{aligned}
& N(\xi, x) \equiv \frac{1}{\pi} \ln \left|\frac{1+\Lambda}{1-\Lambda}\right|, \\
& \Lambda(\xi, x) \equiv\left[\frac{(1-x)(1+\xi)}{(1-\xi)(1+x)}\right]^{1 / 2} .
\end{aligned}
$$

We observe that all terms on the right-hand side of (29) are finite; therefore the integration by parts is legitimate. As $\varepsilon \rightarrow 0$, the sum of the first two terms in (29) approaches zero. It can be shown that the last two integrals converge.

Therefore, the required functional dependence is of the form

$$
2 \varphi^{+}=-\int_{-1}^{1} N(\xi, x) \gamma(\xi, t) \mathrm{d} \xi .
$$

With the help of (11), (13), (30) and (31), we arrive at the expression for the aerodynamic reaction of the fluid. Writing out the dimensionless coordinate scaling factors $\tau$ and $\ell$ explicitly, we have:

$$
\begin{aligned}
q_{f}(x, t) & =p^{-}(x, t)-p^{+}(x, t) \\
& =\rho_{f}\left(\frac{1}{\tau} \frac{\partial}{\partial t}+\frac{1}{\ell} v_{\infty} \frac{\partial}{\partial x}\right)\left(\varphi^{+}(x, t)-\varphi^{-}(x, t)\right) \\
& =\rho_{f}\left(\frac{1}{\tau} \frac{\partial}{\partial t}+\frac{1}{\ell} v_{\infty} \frac{\partial}{\partial x}\right)\left(2 \varphi^{+}(x, t)\right) \\
& =-\rho_{f}\left(\frac{1}{\tau} \frac{\partial}{\partial t}+\frac{1}{\ell} v_{\infty} \frac{\partial}{\partial x}\right) \int_{-1}^{1} N(\xi, x) \gamma(\xi, t) \mathrm{d} \xi \\
& =-\rho_{f}\left(\frac{1}{\tau} \frac{\partial}{\partial t}+\frac{1}{\ell} v_{\infty} \frac{\partial}{\partial x}\right) \int_{-1}^{1} N(\xi, x)\left(\frac{\ell}{\tau} \frac{\partial w}{\partial t}+v_{\infty} \frac{\partial w}{\partial x}\right) \mathrm{d} \xi .
\end{aligned}
$$

The expression (32) is valid, because $N(\xi, x)$ is a Green's function of Laplace's equation, and thus, the improper integral (31) converges (see, e.g., Evans 1998). 
Alternatively, it can be shown directly that the $L_{1}$ norm of $N(\xi, x)$ is finite, from which the convergence of (31) follows. We outline the argument below; for details, see (Banichuk et al., 2008, Appendix B). Assume that $x \in$ $[-1,1]$ and $t \in[0, \infty)$ are fixed. By Hölder's inequality, we have the estimate

$$
\begin{aligned}
\int_{-1}^{1} N(\xi, x) \gamma(\xi, t) \mathrm{d} \xi & \leq \int_{-1}^{1}|N(\xi, x)| \mathrm{d} \xi \cdot \max _{\xi \in[-1,1]}|\gamma(\xi, t)| \\
& \equiv M(t) \cdot \int_{-1}^{1}|N(\xi, x)| \mathrm{d} \xi
\end{aligned}
$$

The factor $M(t)$ is clearly a nonnegative, finite number (for each fixed $t$ ), so it does not affect the convergence. We can omit the absolute value in the integral, because $N(\xi, x) \geq 0$ over the whole domain. Furthermore, the function $N(\xi, x)$ is symmetric with respect to the lines $\xi=x$ and $\xi=-x$. Therefore it is sufficient to prove that the integral $\int_{-1}^{x} N(\xi, x) \mathrm{d} \xi$ converges. It can be shown that $N(\xi, x) \leq 1 / \sqrt{|x-\xi|}$ over the whole domain, and by direct calculation, $\int_{-1}^{x}(1 / \sqrt{x-\xi}) \mathrm{d} \xi=2 \sqrt{1+x}$. The sandwich theorem then establishes the convergence of the original integral.

As a final note for this section, let us construct an added-mass approximation from the present model. Approximate

$$
\int_{-1}^{1} N(\xi, x) f(\xi) \mathrm{d} \xi \approx \mu \int_{-1}^{1} \delta(\xi, x) f(\xi) \mathrm{d} \xi
$$

where $f$ is any function, $\delta$ the Dirac delta distribution, and the constant

$$
\mu \equiv \operatorname{mean}_{x \in(-1,1)} \int_{-1}^{1} N(\xi, x) \mathrm{d} \xi=\frac{1}{2} \int_{-1}^{1}\left[\int_{-1}^{1} N(\xi, x) \mathrm{d} \xi\right] \mathrm{d} x .
$$

Numerically we find that $\mu=\pi / 4$. For simplicity, consider only the inertial terms of (1) and the approximated $q_{f}$, the latter obtained by inserting (33) and the value of $\mu$ into (32). In dimensionless coordinates, we have

$$
\frac{m}{\tau^{2}} \frac{\partial^{2} w}{\partial t^{2}}+2 \frac{m V_{0}}{\ell \tau} \frac{\partial^{2} w}{\partial x \partial t}+\frac{m V_{0}^{2}}{\ell^{2}} \frac{\partial^{2} w}{\partial x^{2}}=-\rho_{f} \frac{\pi}{4}\left[\frac{\ell}{\tau^{2}} \frac{\partial^{2} w}{\partial t^{2}}+2 \frac{v_{\infty}}{\tau} \frac{\partial^{2} w}{\partial x \partial t}+\frac{v_{\infty}^{2}}{\ell} \frac{\partial^{2} w}{\partial x^{2}}\right],
$$

from which we obtain

$$
\frac{1}{\tau^{2}}\left[m+m_{a}\right] \frac{\partial^{2} w}{\partial t^{2}}+2 \frac{V_{0}}{\ell \tau}\left[m+m_{a} r_{v}\right] \frac{\partial^{2} w}{\partial x \partial t}+\frac{V_{0}^{2}}{\ell^{2}}\left[m+m_{a} r_{v}^{2}\right] \frac{\partial^{2} w}{\partial x^{2}}=0,
$$

where $m_{a} \equiv \ell \rho_{f} \pi / 4$ and $r_{v} \equiv v_{\infty} / V_{0}$. This reduces to a classical one- or three-term single-parameter added-mass model by choosing $r_{v}=0$ (i.e. $\left.v_{\infty}=0\right)$ or $r_{v}=1\left(v_{\infty}=V_{0}\right)$. 
The prediction for the added mass $m_{a}$ thus derived agrees with eq. (12a) of Pramila (1986), if we take $\alpha=0.5$ in Pramila's eq. (12a). Note that our $\ell$ denotes the span half-length and Pramila's $a$ denotes the full length. According to Pramila (1986), Table II, the choice $\alpha=0.5$ corresponds to an aspect ratio slightly larger than 1.0 (span slightly longer than wide).

Compare also eq. (13) of Pramila (1987), due to T. Y.-T. Wu, reported to hold for long and narrow spans. Here the corresponding added mass becomes $b \rho_{f} \pi / 4$, when the force per unit area is considered. This formulation, instead of $\ell$, uses the plate width $b$ as the length scale. Comparing to our approximation in (35), $\ell \rho_{f} \pi / 4$, the best agreement is obtained when $\ell / b \approx 1$, i.e. aspect ratio $2 \ell / b \approx 2$.

For the rest of this paper, we will use the original formulation (32) without the added-mass approximation.

\section{The semi-discrete form}

In this section, we apply the Fourier-Galerkin method in order to obtain a form suitable for numerical analysis. We represent the displacement $w$ as a Galerkin series,

$$
w(x, t)=s \sum_{n=1}^{\infty} f_{n}(t) \Psi_{n}(x),
$$

where $s$ is an arbitrary scaling parameter with the dimension $[s]=\mathrm{m}$. The functions $f_{n}$ and $\Psi_{n}$ are both dimensionless. Since the value of $s$ is arbitrary, we will use the particularly convenient choice $s=\ell$.

For the shape functions $\Psi_{n}$, we choose the eigenmodes of free vibrations of a membrane in vacuum,

$$
\Psi_{n}(x) \equiv \sin \left(n \pi \frac{x+1}{2}\right), \quad x \in[-1,1] .
$$

This is a Fourier basis that splits the space component of the solution into vibration modes along the $x$ axis. By its construction, it automatically accounts for the boundary conditions (3).

Let us define the quantity

$$
V_{0 D} \equiv \sqrt{T / m},
$$

which is the critical divergence velocity of a membrane in vacuum (see, e.g., 
Chang and Moretti 1991). Additionally, define the dimensionless quantities

$$
\begin{aligned}
\gamma & \equiv \ell \frac{\rho_{f}}{m}, \\
\beta & \equiv \frac{D}{m V_{0 D}^{2} \ell^{2}}=\frac{D}{\ell^{2} T}, \\
\lambda & \equiv V_{0} / V_{0 D}, \\
\theta & \equiv v_{\infty} / V_{0 D}, \\
\alpha & \equiv \frac{\ell / \tau}{V_{0 D}} .
\end{aligned}
$$

These denote a fluid effect coefficient, dimensionless bending rigidity, dimensionless velocities for the plate and the fluid, and dynamic scale, respectively. The quantities (39)-(42) are defined as in our earlier instability study, Banichuk et al. (2010b); the quantity (43) is new to the dynamic case.

Finally, let us define the dimensionless external load (here spelling out the primes for the sake of clarity)

$$
g^{\prime \prime}\left(x^{\prime}, t^{\prime}\right) \equiv \frac{\ell}{m V_{0 D}^{2}} g^{\prime}\left(x^{\prime}, t^{\prime}\right)=\frac{\ell}{T} g^{\prime}\left(x^{\prime}, t^{\prime}\right)
$$

and the space-discrete load vector, whose $j$ th component is (spelling out the primes)

$$
G_{j}\left(t^{\prime}\right) \equiv \int_{-1}^{1} g^{\prime \prime}\left(x^{\prime}, t^{\prime}\right) \Psi_{j}\left(x^{\prime}\right) \mathrm{d} x^{\prime} .
$$

We divide equation (1) by $m$ and insert (38)-(45). Writing out the dimensionless coordinate scaling factors explicitly, we have

$$
\begin{aligned}
& \frac{1}{\tau^{2}} \frac{\partial^{2} w}{\partial t^{2}}+2 \frac{V_{0}}{\ell \tau} \frac{\partial^{2} w}{\partial x \partial t}+\frac{1}{\ell^{2}}\left(V_{0}^{2}-V_{0 D}^{2}\right) \frac{\partial^{2} w}{\partial x^{2}}+\frac{D}{m \ell^{4}} \frac{\partial^{4} w}{\partial x^{4}} \\
&+\frac{\rho_{f}}{m}\left(\frac{1}{\tau} \frac{\partial}{\partial t}+\frac{1}{\ell} v_{\infty} \frac{\partial}{\partial x}\right) \int_{-1}^{1} N(\xi, x)\left[\frac{\ell}{\tau} \frac{\partial w}{\partial t}(\xi, t)+v_{\infty} \frac{\partial w}{\partial x}(\xi, t)\right] \mathrm{d} \xi \\
&-\frac{g}{m}=0,
\end{aligned}
$$

which can be written more compactly in the form

$$
\mathcal{L}(w)+\frac{\rho_{f}}{m} \mathcal{K}(w)-\frac{g}{m}=0,
$$

where the operators $\mathcal{L}$ and $\mathcal{K}$ are defined by the obvious identifications.

The same caution as in the earlier instability study, Banichuk et al. (2010b), applies here, too. We must be careful with the derivative in front of the integral in the fluid term in (46), because the aerodynamic kernel $N(\xi, x)$ is singular. We cannot directly take the derivative operator into the 
integral, because the $L_{1}$ norm of $\partial N / \partial x$ is not finite. A straightforward, but somewhat lengthy, calculation finds that $\partial N / \partial x$ has singularities of type $1 / x^{\alpha}$, where $\alpha \geq 1$. The singularities are located at $x= \pm 1$ with $\alpha=3 / 2$, and at $x=\xi$ with $\alpha=1$.

However, as was shown, the integral in (31) is absolutely convergent, and thus the function $\varphi^{+}$is bounded. We can choose from two approaches. The first approach is to integrate first, and then differentiate the result by any method. The second approach, more applicable here because we work in the weak form and do not have a closed-form antiderivative, is to integrate by parts against the test function as usual. This is legitimate despite the singularity, because the integrand of the weak form is a product of two bounded, integrable functions.

We now consider the system of equations corresponding to the dynamics of the web, expressed by the weak form of (46). Constructing the weak form using the Galerkin series (36) gives us the following expressions for the operators $\mathcal{L}$ and $\mathcal{K}$ :

$$
\begin{aligned}
\int_{-1}^{1} \mathcal{L}\left(\ell \sum_{n=1}^{\infty} f_{n} \Psi_{n}\right) \Psi_{j} \mathrm{~d} x=\ell \sum_{n=1}^{\infty} & {\left[\frac{\ell}{\tau^{2}} A_{j n} \frac{\mathrm{d}^{2} f_{n}}{\mathrm{~d} t^{2}}+2 \frac{V_{0}}{\tau} B_{j n} \frac{\mathrm{d} f_{n}}{\mathrm{~d} t}\right.} \\
& \left.+\frac{1}{\ell}\left(V_{0}^{2}-V_{0 D}^{2}\right) C_{j n} f_{n}+\frac{D}{m \ell^{3}} D_{j n} f_{n}\right]
\end{aligned}
$$

$$
\begin{aligned}
\int_{-1}^{1} \mathcal{K}\left(\ell \sum_{n=1}^{\infty} f_{n} \Psi_{n}\right) \Psi_{j} \mathrm{~d} x=\ell \sum_{n=1}^{\infty}\left[\frac{\ell^{2}}{\tau^{2}} a_{j n} \frac{\mathrm{d}^{2} f_{n}}{\mathrm{~d} t^{2}}+2 \frac{\ell}{\tau} v_{\infty} b_{j n} \frac{\mathrm{d} f_{n}}{\mathrm{~d} t}\right. \\
\left.+v_{\infty}^{2} c_{j n} f_{n}\right]
\end{aligned}
$$

Note that it was convenient to choose the scaling $s=\ell$ in (36), because this allows us to extract the common factor $\ell$ from the fluid terms (49). This, in turn, justifies the definition (39) for the fluid effect coefficient.

The matrices $A_{j n}, B_{j n}, C_{j n}, D_{j n}, a_{j n}, b_{j n}$ and $c_{j n}$ are defined by 


$$
\begin{aligned}
A_{j n} & =\int_{-1}^{1} \Psi_{n}(x) \Psi_{j}(x) \mathrm{d} x=\delta_{j n} \\
B_{j n} & =\int_{-1}^{1} \frac{\mathrm{d} \Psi_{n}}{\mathrm{~d} x}(x) \Psi_{j}(x) \mathrm{d} x=\left\{\begin{array}{l}
n j \\
\frac{n j}{n^{2}-j^{2}}\left((-1)^{j+n}-1\right), j \neq n
\end{array}\right. \\
C_{j n} & =\int_{-1}^{1} \frac{\mathrm{d}^{2} \Psi_{n}}{\mathrm{~d} x^{2}}(x) \Psi_{j}(x) \mathrm{d} x=-\left(\frac{j \pi}{2}\right)^{2} \delta_{j n} \\
D_{j n} & =\int_{-1}^{1} \frac{\mathrm{d}^{4} \Psi_{n}}{\mathrm{~d} x^{4}}(x) \Psi_{j}(x) \mathrm{d} x=\left(\frac{j \pi}{2}\right)^{4} \delta_{j n} \\
a_{j n} & =\int_{-1}^{1} \int_{-1}^{1} \Psi_{n}(\xi) N(\xi, x) \Psi_{j}(x) \mathrm{d} \xi \mathrm{d} x \\
b_{j n} & =\frac{1}{2}\left(I_{j n}-I_{n j}\right) \text { and } \\
c_{j n} & =-\int_{-1}^{1} \int_{-1}^{1} \frac{\mathrm{d} \Psi_{n}}{\mathrm{~d} x}(\xi) N(\xi, x) \frac{\mathrm{d} \Psi_{j}}{\mathrm{~d} x}(x) \mathrm{d} \xi \mathrm{d} x,
\end{aligned}
$$

where $j, n=1,2,3, \ldots$ and $\delta_{j n}$ is the Kronecker delta. In equation (55),

$$
I_{j n} \equiv \int_{-1}^{1} \int_{-1}^{1} \frac{\mathrm{d} \Psi_{n}}{\mathrm{~d} x}(\xi) N(\xi, x) \Psi_{j}(x) \mathrm{d} \xi \mathrm{d} x
$$

In equation (56), an integration by parts has been carried out. The boundary term vanishes, because $\Psi_{j}( \pm 1)=0$ for all $j$.

If different boundary conditions are used, where $w( \pm 1) \neq 0$, the boundary term needs to be taken into account; see equations (36) and (46). The closed-form solutions of (50)-(53) are specific to the basis (37). In all other respects, the definitions (50)-(56) always hold, regardless of the basis or boundary conditions chosen.

The integrals in (54)-(56) have no closed-form solution, but some useful properties may be obtained analytically, assuming the basis (37). If $j+n$ is odd, then $a_{j n}=c_{j n}=0$ by considering the symmetries of each integrand. The matrix $b_{j n}$ is antisymmetric, and if $j+n$ is even, then $b_{j n}=0$. The matrices $a_{j n}$ and $c_{j n}$ are symmetric by the symmetry of $N(\xi, x)$ with respect to the line $x=\xi$ and the application of Fubini's theorem. When $j+n$ is even, each integrand $a_{j n}$ and $c_{j n}$ is symmetric with respect to the lines $x=\xi$ and $x=-\xi$.

Inserting (48), (49) and (39)-(43) into the weak form of (47), dividing the result by the quantity $V_{0 D}^{2}$, applying (45) and rearranging the terms by 
the order of the time derivatives, we obtain the dimensionless weak form

$$
\begin{aligned}
& \sum_{n=1}^{\infty}\left(\alpha^{2}\left[A_{j n}+\gamma a_{j n}\right]\right. \frac{\mathrm{d}^{2} f_{n}}{\mathrm{~d} t^{2}}+2 \alpha\left[\lambda B_{j n}+\gamma \theta b_{j n}\right] \frac{\mathrm{d} f_{n}}{\mathrm{~d} t} \\
&\left.+\left[\left(\lambda^{2}-1\right) C_{j n}+\gamma \theta^{2} c_{j n}+\beta D_{j n}\right] f_{n}\right)-G_{j}=0,
\end{aligned}
$$

where definitions (39)-(43), (44)-(45), and (50)-(56) have been used. As for the initial conditions, by inserting (36) into (4), using (50) and noting that we chose $s=\ell$, we have

$$
\begin{gathered}
f_{j}(0)=\frac{1}{\ell} \int_{-1}^{1} \Psi_{j}(x) g_{1}(x) \mathrm{d} x, \quad j=1,2,3, \ldots \\
\frac{\mathrm{d} f_{j}}{\mathrm{~d} t}(0)=\frac{1}{\ell} \int_{-1}^{1} \Psi_{j}(x) g_{2}(x) \mathrm{d} x, \quad j=1,2,3, \ldots .
\end{gathered}
$$

Thus the original initial boundary value problem (1), (3)-(4) has been transformed into a Cauchy problem for the system of ordinary differential equations (57) with initial conditions (58)-(59).

The corresponding buckling problem that was investigated in our previous study, Banichuk et al. (2010b), can be obtained from (57) by setting $\alpha=0$ (i.e., $\tau \rightarrow \infty$ ) and $G_{j} \equiv 0$.

\section{Time integration}

Let us truncate the Galerkin series at a given value of $n=n_{0}$. The resulting finite equation corresponding to (57) is a linear, second-order, nonhomogeneous, vector-valued ordinary differential equation with constant coefficients, of the general form

$$
M_{2} \frac{\mathrm{d}^{2} \mathbf{f}(t)}{\mathrm{d} t^{2}}+M_{1} \frac{\mathrm{d} \mathbf{f}(t)}{\mathrm{d} t}+M_{0} \mathbf{f}(t)=\mathbf{G}(t),
$$

where the coefficient matrices are defined by the obvious identifications, and $\mathbf{G}$ is a vector consisting of the components $G_{j}$ defined by (45).

System (60) can be reduced to a twice larger first-order one by standard techniques; let us define

$$
\mathbf{u} \equiv\left[\begin{array}{l}
\mathbf{f}^{\prime} \\
\mathbf{f}
\end{array}\right]
$$


where the prime denotes the time derivative. Taking into account that $M_{2}$ is small enough to invert numerically, the expanded equation system becomes

$$
\frac{\mathrm{d}}{\mathrm{d} t}\left[\begin{array}{l}
\mathbf{f}^{\prime} \\
\mathbf{f}
\end{array}\right]=\left[\begin{array}{cc}
-M_{2}^{-1} M_{1}-M_{2}^{-1} M_{0} \\
I & 0
\end{array}\right]\left[\begin{array}{l}
\mathbf{f}^{\prime} \\
\mathbf{f}
\end{array}\right]+\left[\begin{array}{c}
M_{2}^{-1} \mathbf{G} \\
0
\end{array}\right],
$$

which can be written as

$$
\mathbf{u}^{\prime}=M \mathbf{u}+\mathbf{g}(t)
$$

where

$$
M=\left[\begin{array}{cc}
-M_{2}^{-1} M_{1}-M_{2}^{-1} M_{0} \\
I & 0
\end{array}\right], \quad \mathbf{g}(t)=\left[\begin{array}{c}
M_{2}^{-1} \mathbf{G}(t) \\
0
\end{array}\right] .
$$

Standard time integration techniques, such as the Fourth-order Runge-Kutta method, are directly applicable to the system (63). In this study, a diagonalization method (see, e.g., Kreyszig 1993) was used, taking advantage of the small size of the equation system.

For convenience, let us briefly review the diagonalization method. To diagonalize the system (63), we assume that $M$ has a full eigenvector basis (in practice, this holds). Define $\Lambda, X, \mathbf{z}$, and $\mathbf{h}(t)$ :

$$
\Lambda \equiv X^{-1} M X, \quad \mathbf{u} \equiv X \mathbf{z}, \quad X \mathbf{h}(t) \equiv \mathbf{g}(t),
$$

where $\Lambda$ is a diagonal matrix with the (complex) eigenvalues $\lambda_{j}$ of $M$ on the diagonal, and $X$ is a unitary matrix containing the eigenvectors of $M$ in its columns. Using the relations (65), equation (63) becomes

$$
\mathbf{z}^{\prime}=\Lambda \mathbf{z}+\mathbf{h}(t),
$$

The solution for the $j$ th component of $\mathbf{z}$ is (Kreyszig 1993, p. 191)

$$
z_{j}(t)=e^{\lambda_{j} t}\left[z_{j}(0)+\int_{\hat{t}=0}^{t} e^{-\lambda_{j} \hat{t}} h_{j}(\hat{t}) \mathrm{d} \hat{t}\right],
$$

where $\hat{t}$ is a dummy variable for integration and $j=1,2, \ldots, 2 \cdot n_{0}$. The initial value $\mathbf{z}(0)$ is evaluated by using (58)-(59) and (61), and solving the linear system in (65) for $\mathbf{z}$. Using (67), (65) and (61), the space-discrete solution $\mathbf{f}(t)$ and its time derivative $\mathbf{f}^{\prime}(t)$ may be computed at any desired time $t$ without the need for timestepping.

Although the eigenvalues $\lambda_{j}$ are in general complex, the solution $\mathbf{u}$ stays real-valued for real-valued initial data. Because the equation system is small, and $M$ is constant in time, it is not expensive to compute $\Lambda$ and $X$ using a standard numerical eigenvalue solver. 
We conclude this section with a note on the dynamic instability analysis of Bolotin (1963). The flutter problem for our space-discrete system is as follows. In (60), let $f(t)=F e^{s t}$, where $F$ is a constant vector. We wish to find all $s \in \mathbb{C}$ (and optionally, also nontrivial $F \in \mathbb{R}^{n_{0}}$ ) such that

$$
\left[\left(M_{2} s^{2}+M_{1} s+M_{0}\right) F\right] e^{s t} \equiv L(s) F e^{s t}=0 .
$$

To find $s$, we use the determinant method. It is easily seen that the zeroes of $\operatorname{det}\left(L(s)\right.$ ) (as a function of $s$ ) are the eigenvalues of $M$, the $2 \cdot n_{0} \times 2 \cdot n_{0}$ matrix defined by equation (64). Expand $\operatorname{det}\left(M-\lambda_{j} I\right)$ as a block determinant, noting that the necessary blocks commute. Multiply (68) from the left by $M_{2}^{-1}$. Compare the zero determinant conditions to find that $s=\lambda_{j}$.

This gives us the eigenfrequency response of the system, e.g., for various values of $V_{0}$ with the other parameters kept fixed. The critical velocity at which $s_{j}=0$ for all $j=1,2, \ldots, 2 \cdot n_{0}$, obtained by a numerical search procedure, matches the critical velocity predicted by the static instability analysis (Banichuk et al., 2010b).

\section{Numerical results}

In our computations, the Galerkin series was truncated at $n_{0}=56$. Physical parameters were chosen as typical for a paper web surrounded by air: $T=500 \mathrm{~N} / \mathrm{m}, m=80 \mathrm{~g} / \mathrm{m}^{2}, \rho_{f}=1.25 \mathrm{~kg} / \mathrm{m}^{3}$, and $h=10^{-4} \mathrm{~m}$. For the elastic parameters, an isotropic approximation was used with $v=0.3$ and $E=10^{9} \mathrm{~N} / \mathrm{m}^{2}$. The mass per unit area of the web was $m=0.08 \mathrm{~kg} / \mathrm{m}^{2}$. For the span half-length, the value $\ell=1 \mathrm{~m}$ was used. The timescale parameter was chosen as $\tau=\ell / V_{0 D}$, which leads to $\alpha=1$ (equation (43)). Recall that the cylindrical deformation assumption for travelling plates is acceptable if the span is long and narrow (Banichuk et al., 2010b). The results should be viewed with this in mind.

Except for a few trivial special cases, the load term needs to be integrated numerically. This can be done to any desired precision using standard methods. Thus the choice of the points of time at which to compute the solution does not affect the accuracy of the method. There is, as in all numerical time integration methods, a source of cumulative error. Here it stems from the numerical accuracy of the eigenvalue solver used to compute the eigenvalues and eigenvectors used for diagonalization. As can be seen from equation (67), the transformed solution $\mathbf{z}$ will drift away from the exact one at a rate which depends on the amount of numerical error in the eigenvalues. The error in the solution $\mathbf{u}$ then behaves as a linear combination of these errors, by the transformation (65). The error is independent of the spacing of the points of time at which the solution is evaluated. 
The diagonalization method does not cause numerical dissipation of energy, because the qualitative behaviour of the first-order system (66) is captured by (67). However, numerical error in the eigenvalues may cause small imaginary components to be introduced into the Galerkin coefficients $\mathbf{u}$, which are known a priori to be real. This can be avoided by inspecting the imaginary part of the solution at each point of time for which it is computed, and then either ignoring the imaginary part (if small) or stopping computation. In our tests, to keep the computation consistent, we chose to test for imaginary parts in the original solution vector, but discard them only in visualization. The validity criterion used was $\operatorname{Im}\left(u_{j}\right)<10^{-8}$ separately for each component $j=1,2, \ldots, 2 \cdot n_{0}$. In practice, this criterion was never violated.

Two kinds of results were computed, direct temporal simulations and lowest eigenfrequency behaviour. The velocities $V_{0}$ and $v_{\infty}$ were varied across the studied cases.

Some direct simulations are shown in Figure 2. Each simulation is presented as a figure consisting of three parts. The top half displays a spacetime plot of the displacement function $w(x, t)$. The horizontal axis represents dimensionless time $t$ and the vertical axis designates the position $x$ between the rollers at $x= \pm 1$ (note the orientation, positive $x$ up). The shade of each point in the image indicates the height, measured from zero displacement.

The bottom half of each figure is made up of two graphs. The graph on the left shows the displacement of the plate as a function of $x$ at a few selected times $t$. The graph on the right shows the time behaviour of the centre point of the plate $w(0, t)$. The corresponding points in the lower two graphs are marked with circles.

All computations were performed with a configuration where the initial position of the plate was given. For the cases in Figure 2, the initial condition for position was

$$
w(x, 0) \equiv g_{1}(x) \equiv a \cdot \sin \left(\pi \frac{x+1}{2}\right),
$$

where the initial amplitude at the center point was $a=5 \cdot 10^{-3}$. The initial transverse velocity was zero,

$$
\frac{\partial w}{\partial t}(x, 0) \equiv g_{2}(x) \equiv 0,
$$

and there were no external disturbances,

$$
g(x, t) \equiv 0 .
$$


In the first row of Figure 2, we have as a fundamental test case a plate in vacuum, travelling at various speeds and undergoing a steady, cylindrical vibration. In the space-time plot, the shapes are aligned at an angle to the $x$ axis. Due to the axial motion toward the positive $x$ direction, the positive- $x$ half of the plate experiences each maximum (minimum) of the vibration before the negative- $x$ half does. Physically, as is well known, the wave travelling to the direction of travel on an axially moving medium moves at a higher velocity than the wave travelling in the opposite direction. Mathematically, the phenomenon can be seen as the velocity-dependent phase shift in the eigenmodes of axially travelling strings and beams that was discussed by Wickert and Mote (1990). As was noted in the problem setup, the flat panel model shares the mathematical formulation with the beam model, so we can expect the same phenomenon to occur.

Let us now move onto the focus of the present study, and consider the effect of fluid-structure interaction. The rest of the rows in Figure 2 represent the dynamic response of the plate in stationary and in axially moving fluid. The qualitative behaviour in our Figure 2 is seen to be similar to Figure 2 in Chang et al. (1991), where a free vibration cycle of a travelling threadline from a direct simulation was plotted.

Figure 3 shows the behaviour of the nondimensional first natural frequency against the nondimensional velocity of the plate, when submerged in ideal fluid. Two cases are shown: stationary air $\left(v_{\infty}=0\right)$, and the whole air mass moving with the web $\left(v_{\infty}=V_{0}\right)$. The figure was produced by numerically solving the eigenvalues of $M$ as explained above, and then picking the eigenvalue with the smallest imaginary part at each value of $V_{0}$.

The normalization used in Figure 3 for the frequency axis is the first natural frequency of a stationary plate undergoing cylindrical deformation in vacuum, and the velocity is normalized by the critical velocity in vacuum (both of these numerically computed, with $q_{f}(x, t) \equiv 0$ and all problem parameters the same). It can be seen that the presence of fluid decreases the first natural frequency, as expected (see Pramila, 1986, and Kulachenko et al., 2007b).

Three pairs of classical analytical results from two added-mass models from a study by Pramila (1987) are included in Figure 3 for comparison, plotted for our problem parameters. Each pair begins at a single point at $V_{0}=0$, and the different pairs correspond to different aspect ratios (AR, span length per width). The added masses are constants, which are affected by $\beta_{\operatorname{Pr}}$, the $\beta$ value of Pramila (1986), Table II, which depends on the AR. For the results shown here, this dependence was modelled in the form AR = $\operatorname{AR}\left(\beta_{\operatorname{Pr}}\right)=c_{1} / \beta_{\operatorname{Pr}}+c_{2} / \beta_{\operatorname{Pr}}^{2}$. Performing a least-squares fit to the tabulated data, the values $c_{1}=0.1387$ and $c_{2}=0.5318$ were obtained. Alternative forms, with just the first term $1 / \beta_{\mathrm{Pr}}$, and a three-term form including $1 / \beta_{\mathrm{Pr}}^{3}$, were also tested, and the two-term form given here was found to produce 
the most satisfactory fit.

The curve marked as eq. (16) corresponds to an added-mass model modifying all three masses (local inertia, Coriolis and centrifugal), while the one labelled as eq. (22) corresponds to a model modifying only the local inertia mass. For the eq. (16) model, all three added masses are equal. Note that Pramila used mass per unit length in his study (Pramila, 1987), whereas we use mass per unit area. Because the frequency expressions contain only ratios of the parameters, the width can be cancelled out and the results are directly comparable, once the span length and time scaling are taken into account.

Our model at $v_{\infty}=0$ agrees closely with the eq. (22) one, as expected. From the discretization (57) we see that in this case, only the matrix $a_{j n}$ has an effect. This corresponds to a first approximation to a local inertia mass increase. The magnitude of the decrease in eigenfrequency is similar to that reported by Pramila (about 75\% according to Pramila 1986). For a certain aspect ratio $\left(\mathrm{AR} \approx 3.1989, \beta_{\mathrm{Pr}}=0.43\right)$, the predictions coincide for the problem parameters used.

In the case $v_{\infty}=V_{0}$, the models qualitatively agree. If we again use $\beta_{\operatorname{Pr}}=0.43$ for Pramila's model, we see that the prediction given by our model for the critical velocity is approximately $60 \%$ higher than that from the corresponding added-mass approach (Pramila's eq. (16)). On the other hand, if the value $\beta_{\operatorname{Pr}}=0.18$ (corresponding to $\mathrm{AR} \approx 17.1850$ ) is used, then the predictions of critical velocity agree, but the eigenfrequency given by the added-mass model is approximately $60 \%$ higher. Values of $\beta_{\operatorname{Pr}}$ between these two cases produce results that vary continuously from the first case to the second. See Figure 3 for an example. It is seen that the primary difference between the present functional approach and the classical results being compared, as far as the lowest eigenfrequency is concerned, is that the predictions change in the case where all three inertia terms are modified. The aspect ratio never explicitly enters this model, so which prediction differs more, depends on the aspect ratio.

This effect has a simple mathematical explanation. In Pramila's eq. (16), changing the added mass changes the scaling of both axes equally. For each pair of added-mass curves in Figure 3, it is seen that the axis intersection points of the curve corresponding to Pramila's eq. (16) are equal. The present model does not make any such assumption, and thus the scalings, which arise naturally by solving the integro-differential equation, may be different.

Note that equal scaling is not an inherent limitation of the addedmass approach, but is due to the specific form of Pramila's eq. (16). See Chang et al. (1991) for a discussion on how different (but still constant) added masses in each term affect the eigenfrequency behaviour. For an ap- 


$$
\begin{array}{ccc}
\text { Vacuum, } V_{0}=0 \mathrm{~m} / \mathrm{s} & \text { Vacuum, } V_{0}=30 \mathrm{~m} / \mathrm{s} & \text { Vacuum, } V_{0}=60 \mathrm{~m} / \mathrm{s} \\
\text { Insert figure 2a here } & \text { Insert figure } \mathbf{2 b} \text { here } & \text { Insert figure 2c here } \\
v_{\infty}=0 \mathrm{~m} / \mathrm{s}, V_{0}=0 \mathrm{~m} / \mathrm{s} & v_{\infty}=0 \mathrm{~m} / \mathrm{s}, V_{0}=30 \mathrm{~m} / \mathrm{s} & v_{\infty}=0 \mathrm{~m} / \mathrm{s}, V_{0}=60 \mathrm{~m} / \mathrm{s} \\
\text { Insert figure } 2 \mathbf{d} \text { here } & \text { Insert figure } 2 \mathbf{e} \text { here } & \text { Insert figure } 2 \mathbf{f} \text { here } \\
v_{\infty}=+10 \mathrm{~m} / \mathrm{s}, V_{0}=0 \mathrm{~m} / \mathrm{s} & v_{\infty}=+10 \mathrm{~m} / \mathrm{s}, V_{0}=30 \mathrm{~m} / \mathrm{s} & v_{\infty}=+10 \mathrm{~m} / \mathrm{s}, V_{0}=60 \mathrm{~m} / \mathrm{s} \\
\text { Insert figure } 2 \mathbf{g} \text { here } & \text { Insert figure } \mathbf{2 h} \text { here } & \text { Insert figure } 2 \mathbf{i} \text { here } \\
v_{\infty}=-10 \mathrm{~m} / \mathrm{s}, V_{0}=0 \mathrm{~m} / \mathrm{s} & v_{\infty}=-10 \mathrm{~m} / \mathrm{s}, V_{0}=30 \mathrm{~m} / \mathrm{s} & v_{\infty}=-10 \mathrm{~m} / \mathrm{s}, V_{0}=60 \mathrm{~m} / \mathrm{s} \\
\text { Insert figure } 2 \mathbf{j} \text { here } & \text { Insert figure } \mathbf{2 k} \text { here } & \text { Insert figure } 2 \mathbf{1} \text { here }
\end{array}
$$

Figure 2. Dynamic response of the plate with various fluid and plate velocities. First row: vacuum case for comparison.

proach utilizing boundary layer theory to compute added masses as functions of $x$, also resulting in different scalings for the axes, see Frondelius et al. (2006).

In addition to the two classical cases presented in Figure 3, our model opens the possibility for studying the problem with an arbitrary axial flow velocity for the surrounding air. In Figure 4, eigenfrequency curves similar to those in Figure 3 are shown for several different fluid velocities $v_{\infty}$. The normalization procedure is the same as in Figure 3. Note that as is evident from Figure 4, if $v_{\infty}$ is nonzero and independent of $V_{0}$, the eigenfrequency curves are no longer symmetric with respect to $V_{0}=0$.

We conclude this section with a direct simulation of a special case. The solutions shown in Figure 2 are periodic and stable. Figure 5 represents the limiting case where a nontrivial static solution (divergence) exists. For this case, the starting position of the plate was specified as the critical eigenmode of the corresponding static instability problem. The static problem was solved as reported in Banichuk et al. (2010b), and the obtained numerical Fourier-Galerkin coefficients and the critical velocity were used as input data for the dynamic case. In this configuration, the initial transverse velocity was zero as per equation (70), and there were no external disturbances, as per equation (71). The computed solution stays constant in time, as expected. 


\section{Insert Figure 3 here}

Figure 3. Behaviour of the nondimensional first natural frequency as a function of the nondimensional velocity of the plate. Normalized by the axis intersecting values in vacuum. For the upper solid line, fluid velocity $v_{\infty}=0$. For the lower solid line, $v_{\infty}=V_{0}$, i.e. the air mass moves with the web. For comparison, the vacuum case (dashed line) and three pairs of results corresponding to Pramila's added-mass formulas (dash-dot lines) from the study Pramila (1987) are shown. Each pair begins at a single point at $V_{0}=0$. The different pairs correspond to different aspect ratios (AR, length per width). From top to bottom, $\mathrm{AR} \approx 17.1850$ $\left(\beta_{\mathrm{Pr}}=0.18\right) ; \mathrm{AR} \approx 5.1064\left(\beta_{\mathrm{Pr}}=0.3275\right) ;$ and $\mathrm{AR} \approx 3.1989\left(\beta_{\mathrm{Pr}}=0.43\right.$; in this particular case, the upper curves coincide). The symbol $\beta_{\operatorname{Pr}}$ refers to the $\beta$ value of Pramila (1986), Table II.

\section{Insert Figure 4 here}

Figure 4. Behaviour of the nondimensional first natural frequency as a function of the nondimensional velocity of the plate. Normalized by the axis intersecting values in vacuum. The vacuum case is partly shown for comparison (dash-dot line). Solid lines indicate $v_{\infty} \geq 0$, dashed lines $v_{\infty}<0$. From top to bottom, the absolute values of the fluid velocities are 0 (corresponds to upper solid line of Fig. 3), $10(\theta \approx 0.13), 15(\theta \approx 0.19), 20(\theta \approx 0.25), 25(\theta \approx 0.32)$, and $30 \mathrm{~m} / \mathrm{s}(\theta \approx 0.38)$. Note the scaling of the vertical axis.

\section{Insert Figure 5 here}

Figure 5. Divergence instability, i.e., steady state solution. $V_{0}=70.5257 \mathrm{~m} / \mathrm{s}$. $v_{\infty}=-15 \mathrm{~m} / \mathrm{s}, t_{\text {fin }}=1 \mathrm{~s}, g_{1}(x)$ set to critical eigenmode, $g(x, t) \equiv 0, g_{2}(x) \equiv 0$.

\section{Conclusion}

In this paper, an analytical functional representation was derived for the aerodynamic reaction of axially moving ideal fluid on the surface of a travelling vibrating plate, under the assumption of cylindrical deformation (flat panel model). This assumption is reasonably valid for long, narrow strips. The present study can be viewed as a generalization of earlier studies that have allowed for either the movement of the surrounding fluid or the plate (or beam) only, see e.g. Kornecki et al. (1976). It is also a generalization of our previous study, Banichuk et al. (2010b), in which the static instability behaviour of this system was investigated.

Using the obtained functional representation, dynamic vibration analysis was reduced to the solution of a fourth-order integro-differential equation. The problem was studied with simply supported (pinned) boundary conditions. However, the solution from the performed fluid-structure interaction analysis is applicable for any boundary conditions for the ends of the moving plate. The Fourier-Galerkin method and a diagonalization method were employed for the numerical solution of the obtained integrodifferential equation. The presented discretized solution can be fairly easily 
adapted to other Galerkin bases, or to cases with different boundary conditions.

Both direct temporal simulations and an eigenfrequency analysis were performed. In the direct simulations, the initial position of the plate was specified. There were no external disturbances and the initial transverse velocity of the plate was zero. The model also allows for nonzero initial transverse velocity, and for specifying an external disturbance function.

The behaviour of the lowest eigenfrequency of the plate was investigated at several different axial fluid velocities. The classical cases of stationary air and the air mass moving with the plate were included, as were also examples of arbitrary axial fluid velocities made possible by the present approach. It was observed that the presence of fluid, in all cases, significantly reduces the first natural frequency when compared to the vacuum case. This matches known results; see, e.g., Kulachenko et al. (2007b) and Pramila (1986).

The results of the lowest eigenfrequency analysis were seen to qualitatively agree with the classical added-mass results of Pramila (1987). A significant quantitative difference either in the critical velocity or in the lowest eigenfrequency was observed in the case where the air mass is assumed to move with the plate, depending on the aspect ratio of the plate used in the added-mass model. It was seen that this effect is due to a difference in how mass is handled in the added-mass model, when compared to the present model.

Finally, at the divergence velocity and shape predicted by static analysis (Banichuk et al., 2010b), a direct dynamic simulation confirmed the steady-state property of the solution.

The present research raises some questions, which require further studies to explore. The sensitivity of the physical model on small changes in its parameters is an important question, for which a separate parametric study is needed. Also a more complete modal analysis, not limited to the first eigenfrequency but including higher frequencies and also the corresponding modes, remains a direction for future research.

The investigation of ideal models provides a solid foundation for more complex multiphysics problems. The model developed can, with certain limitations, describe the dynamic behaviour of a moving paper web interacting with axially moving air. Thus, we used for numerical computations the parameter values corresponding to air and some paper materials.

Acknowledgement: This research was supported by the MASI Tekes Technology Programme and the Ellen and Artturi Nyyssönen Foundation (EANS). 


\section{References}

Archibald, F. R., Emslie, A. G., 1958. The vibration of a string having a uniform motion along its length. ASME Journal of Applied Mechanics 25, 347-348.

Ashley, H., Landahl, M., 1985. Aerodynamics of wings and bodies. Dover.

Banichuk, N., Jeronen, J., Neittaanmäki, P., Tuovinen, T., 2008. Instability analysis for traveling membranes and plates interacting with surrounding ideal fluid. Tech. Rep. 13, University of Jyväskylä.

Banichuk, N., Jeronen, J., Neittaanmäki, P., Tuovinen, T., 2010a. On the instability of an axially moving elastic plate. International Journal of Solids and Structures $47(1), 91-99$.

URL http://dx.doi .org/10.1016/j.ijsolstr. 2009.09.020

Banichuk, N., Jeronen, J., Neittaanmäki, P., Tuovinen, T., 2010b. Static instability analysis for travelling membranes and plates interacting with axially moving ideal fluid. Journal of Fluids and Structures 26 (2), 274291.

URL http://dx.doi .org/10.1016/j.jfluidstructs.2009.09.006

Bisplinghoff, R. L., Ashley, H., 1962. Principles of Aeroelasticity. Dover Publications, Inc., New York, 2nd edition 1975.

Bolotin, V. V., 1963. Nonconservative Problems of the Theory of Elastic Stability. Pergamon Press, New York.

Canuto, C., Hussaini, M. Y., Quarteroni, A., Zang, T. A., 1988. Spectral Methods in Fluid Dynamics. Springer-Verlag, New York, ISBN 3-540-17371-4.

Chang, Y. B., Fox, S. J., Lilley, D. G., Moretti, P. M., 1991. Aerodynamics of moving belts, tapes and webs. Vol. 36. pp. 33-40, presented in ASME Symposium on Dynamics of Axially Moving Continua, Miami, Florida, September 22-25, 1991.

Chang, Y. B., Moretti, P. M., 1991. Interaction of fluttering webs with surrounding air. TAPPI Journal.

Chonan, S., 1986. Steady state response of an axially moving strip subjected to a stationary lateral load. Journal of Sound and Vibration 107, 155-165.

Christou, M. A., Christov, C. I., 2007. Fourier-galerkin method for 2d solitons of boussinesq equation. Mathematics and Computers in Simulation 74, 82-92.

Doaré, O., 2010. Dissipation effect on local and global stability of fluidconveying pipes. Journal of Sound and Vibration 329 (1), 72-83.

Doaré, O., Sauzade, M., Eloy, C., 2011. Flutter of an elastic plate in a channel flow: Confinement and finite-size effects. Journal of Fluids and Structures 27 (1), 76-88.

URL http://dx.doi.org/10.1016/j.jfluidstructs.2010.09.002

Dugundji, J., Dowell, E., Perkin, B., 1963. Subsonic flutter of panels on continuous elastic foundations. AIAA Journal 1 (5), 1146-1154.

Eloy, C., Souilliez, C., Schouveiler, L., 2007. Flutter of a rectangular plate. Journal of Fluids and Structures 23 (6), 904-919. 
Evans, L. C., 1998. Partial Differential Equations. American Mathematical Society, ISBN 0-8218-0772-2.

Firouz-Abadi, R., Noorian, M., Haddadpour, H., 2010. A fluid-structure interaction model for stability analysis of shells conveying fluid. Journal of Fluids and Structures 26 (5), 747-763.

URL http://dx.doi.org/10.1016/j.jfluidstructs.2010.04.003

Frondelius, T., Koivurova, H., Pramila, A., 2006. Interaction of an axially moving band and surrounding fluid by boundary layer theory. Journal of Fluids and Structures 22 (8), 1047-1056.

Garziera, R., Amabili, M., 2000. Damping effect of winding on the lateral vibrations of axially moving tapes. ASME Journal of Vibration and Acoustics 122, 49-53.

Gorman, D. J., 1982. Free Vibration Analysis of Rectangular Plates. Elsevier North Holland, Inc., ISBN 0-444-00601-X.

Guo, C. Q., Païdoussis, M. P., 2000. Stability of rectangular plates with free side-edges in two-dimensional inviscid channel flow. ASME Journal of Applied Mechanics 67, 171-176.

Howell, R., Lucey, A., Pitman, M., 2011. The effect of inertial inhomogeneity on the flutter of a cantilevered flexible plate. Journal of Fluids and Structures 27 (3), 383-393.

URL http://dx.doi.org/10.1016/j.jfluidstructs.2010.11.014

Koivurova, H., Pramila, A., 1997. Nonlinear vibrations of axially moving membrane by finite element method. Computational Mechanics 20, 573581.

Kornecki, A., Dowell, E. H., O'Brien, J., 1976. On the aeroelastic instability of two-dimensional panels in uniform incompressible flow. Journal of Sound and Vibration 47 (2), 163-178.

Kreyszig, E., 1993. Advanced Engineering Mathematics, 7th Edition. John Wiley \& Sons, ISBN 0-471-59989-1.

Kulachenko, A., Gradin, P., Koivurova, H., 2007a. Modelling the dynamical behaviour of a paper web. Part I. Computers \& Structures 85, 131-147.

Kulachenko, A., Gradin, P., Koivurova, H., 2007b. Modelling the dynamical behaviour of a paper web. Part II. Computers \& Structures 85, 148-157.

Kurki, M., Juppi, K., Ryymin, R., Taskinen, P., Pakarinen, P., 1995. On the web tension dynamics on an open draw. In: Proceedings of the Third International Conference on Web Handling. Web Handling Research Center, Oklahoma State University, Stillwater, Oklahoma, pp. 230-244.

Lin, C. C., Mote, C. D., 1995. Equilibrium displacement and stress distribution in a two-dimensional, axially moving web under transverse loading. ASME Journal of Applied Mechanics 62, 772-779.

Miranker, W. L., 1960. The wave equation in a medium in motion. IBM Journal of Research and Development 4, 36-42.

Mote, C. D., 1968. Divergence buckling of an edge-loaded axially moving band. International Journal of Mechanical Sciences 10, 281-195.

Mote, C. D., 1972. Dynamic stability of axially moving materials. Shock and 
Vibration Digest 4 (4), 2-11.

Mote, C. D., 1975. Stability of systems transporting accelerating axially moving materials. ASME Journal of Dynamic Systems, Measurement, and Control, 96-98.

Niemi, J., Pramila, A., 1986. Vibration analysis of an axially moving membrane immersed into ideal fluid by fem. Tech. rep., Tampere.

Païdoussis, M. P., 1998. Fluid-Structure Interactions: Slender Structures and Axial Flow. Vol. 1. Academic Press, ISBN 0-12-544360-9.

Païdoussis, M. P., 2004. Fluid-Structure Interactions: Slender Structures and Axial Flow. Vol. 2. Elsevier Academic Press, ISBN 0-12-544361-7.

Païdoussis, M. P., 2008. The canonical problem of the fluid-conveying pipe and radiation of the knowledge gained to other dynamics problems across applied mechanics. Journal of Sound and Vibration 310, 462-492.

Pramila, A., 1986. Sheet flutter and the interaction between sheet and air. TAPPI Journal 69 (7), 70-74.

Pramila, A., 1987. Natural frequencies of a submerged axially moving band. Journal of Sound and Vibration 113 (1), 198-203.

Sathe, S., Benney, R., Charles, R., Doucette, E., Miletti, J., Senga, M., Stein, K., Tezduyar, T., 2007. Fluid-structure interaction modeling of complex parachute designs with the space-time finite element techniques. Computers \& Fluids 36 (1), 127-135, Challenges and Advances in Flow Simulation and Modeling.

URL http://dx.doi .org/10.1016/j.compfluid.2005.07.010

Shen, J. Y., Sharpe, L., McGinley, W. M., Jan. 1995. Identification of dynamic properties of plate-like structures by using a continuum model. Mechanics Research Communications 22 (1), 67-78.

Sherman, D. I., 1952. On the stress distribution in partitions, an elastic heavy medium which is weakened by elliptic holes. Izvestiya Akademii Nauk SSSR, Otdelenie Tekhnicheskikh Nauk (OTN) 7, 992-1010.

Shin, C., Chung, J., Kim, W., Sep. 2005. Dynamic characteristics of the outof-plane vibration for an axially moving membrane. Journal of Sound and Vibration 286 (4-5), 1019-1031.

Silberman, I., Feb. 1954. Planetary waves in the atmosphere. Journal of Meteorology 11, 27-34.

Simpson, A., 1973. Transverse modes and frequencies of beams translating between fixed end supports. Journal of Mechanical Engineering Science 15, 159-164.

Stein, K., Benney, R., Kalro, V., Tezduyar, T. E., Leonard, J., Accorsi, M., Oct. 2000. Parachute fluid-structure interactions: 3-d computation. Computer Methods in Applied Mechanics and Engineering 190 (3-4), 373-386.

Stein, K., Benney, R., Tezduyar, T., Potvin, J., Dec. 2001. Fluid-structure interactions of a cross parachute: numerical simulation. Computer Methods in Applied Mechanics and Engineering 191 (6-7), 673-687.

Sugiyama, Y., Langthjem, M. A., 2007. Physical mechanism of the destabilizing effect of damping in continuous non-conservative dissipative sys- 
tems. International Journal of Nonlinear Mechanics 42 (1), 132-145.

Swope, R. D., Ames, W. F., 1963. Vibrations of a moving threadline. Journal of the Franklin Institute 275, 36-55.

Sygulski, R., Jan. 2007. Stability of membrane in low subsonic flow. International Journal of Non-Linear Mechanics 42 (1), 196-202.

Tadmor, E., 1987. Stability analysis of finite-difference, pseudospectral and fourier-galerkin approximations for time-dependent problems. SIAM Review 29 (4), 525-555.

Timoshenko, S. P., Woinowsky-Krieger, S., 1959. Theory of plates and shells, 2nd Edition. New York : Tokyo : McGraw-Hill, ISBN 0-07-085820-9.

Ulsoy, A. G., Mote, C. D., 1980. Analysis of bandsaw vibration. Wood Science 13, 1-10.

Ulsoy, A. G., Mote, C. D., 1982. Vibration of wide band saw blades. ASME Journal of Engineering for Industry 104, 71-78.

Wang, X., Jan. 2003. Instability analysis of some fluid-structure interaction problems. Computers \& Fluids 32 (1), 121-138.

Watanabe, Y., Isogai, K., Suzuki, S., Sugihara, M., 2002. A theoretical study of paper flutter. Journal of Fluids and Structures 16 (4), 543-560.

Wickert, J. A., Mote, C. D., 1990. Classical vibration analysis of axially moving continua. ASME Journal of Applied Mechanics 57, 738-744.

$\mathrm{Wu}, \mathrm{X} .$, Kaneko, S., 2005. Linear and nonlinear analyses of sheet flutter induced by leakage flow. Journal of Fluids and Structures 20,927-948. 


\section{Figure captions}

Figure 1:

Travelling plate undergoing cylindrical deformation, supported at $x=-\ell$ and $x=+\ell$, and interacting with air. At infinity, the air moves along the $x$ axis with velocity $v_{\infty}$.

\section{Figure 2:}

Dynamic response of the plate with various fluid and plate velocities. First row: vacuum case for comparison.

\section{Figure 3:}

Behaviour of the nondimensional first natural frequency as a function of the nondimensional velocity of the plate. Normalized by the axis intersecting values in vacuum. For the upper solid line, fluid velocity $v_{\infty}=0$. For the lower solid line, $v_{\infty}=V_{0}$, i.e. the air mass moves with the web. For comparison, the vacuum case (dashed line) and three pairs of results corresponding to Pramila's added-mass formulas (dash-dot lines) from the study Pramila (1987) are shown. Each pair begins at a single point at $V_{0}=0$. The different pairs correspond to different aspect ratios (AR, length per width). From top to bottom, $\mathrm{AR} \approx 17.1850\left(\beta_{\operatorname{Pr}}=0.18\right) ; \mathrm{AR} \approx 5.1064\left(\beta_{\operatorname{Pr}}=0.3275\right)$; and $\mathrm{AR} \approx 3.1989\left(\beta_{\operatorname{Pr}}=0.43\right.$; in this particular case, the upper curves coincide). The symbol $\beta_{\text {Pr }}$ refers to the $\beta$ value of Pramila (1986), Table II.

\section{Figure 4:}

Behaviour of the nondimensional first natural frequency as a function of the nondimensional velocity of the plate. Normalized by the axis intersecting values in vacuum. The vacuum case is partly shown for comparison (dash-dot line). Solid lines indicate $v_{\infty} \geq 0$, dashed lines $v_{\infty}<0$. From top to bottom, the absolute values of the fluid velocities are 0 (corresponds to upper solid line of Fig. 3), $10(\theta \approx 0.13), 15(\theta \approx 0.19), 20(\theta \approx 0.25), 25$ $(\theta \approx 0.32)$, and $30 \mathrm{~m} / \mathrm{s}(\theta \approx 0.38)$. Note the scaling of the vertical axis.

\section{Figure 5:}

Divergence instability, i.e., steady state solution. $V_{0}=70.5257 \mathrm{~m} / \mathrm{s} . v_{\infty}=$ $-15 \mathrm{~m} / \mathrm{s}, t_{\mathrm{fin}}=1 \mathrm{~s}, g_{1}(x)$ set to critical eigenmode, $g(x, t) \equiv 0, g_{2}(x) \equiv 0$. 

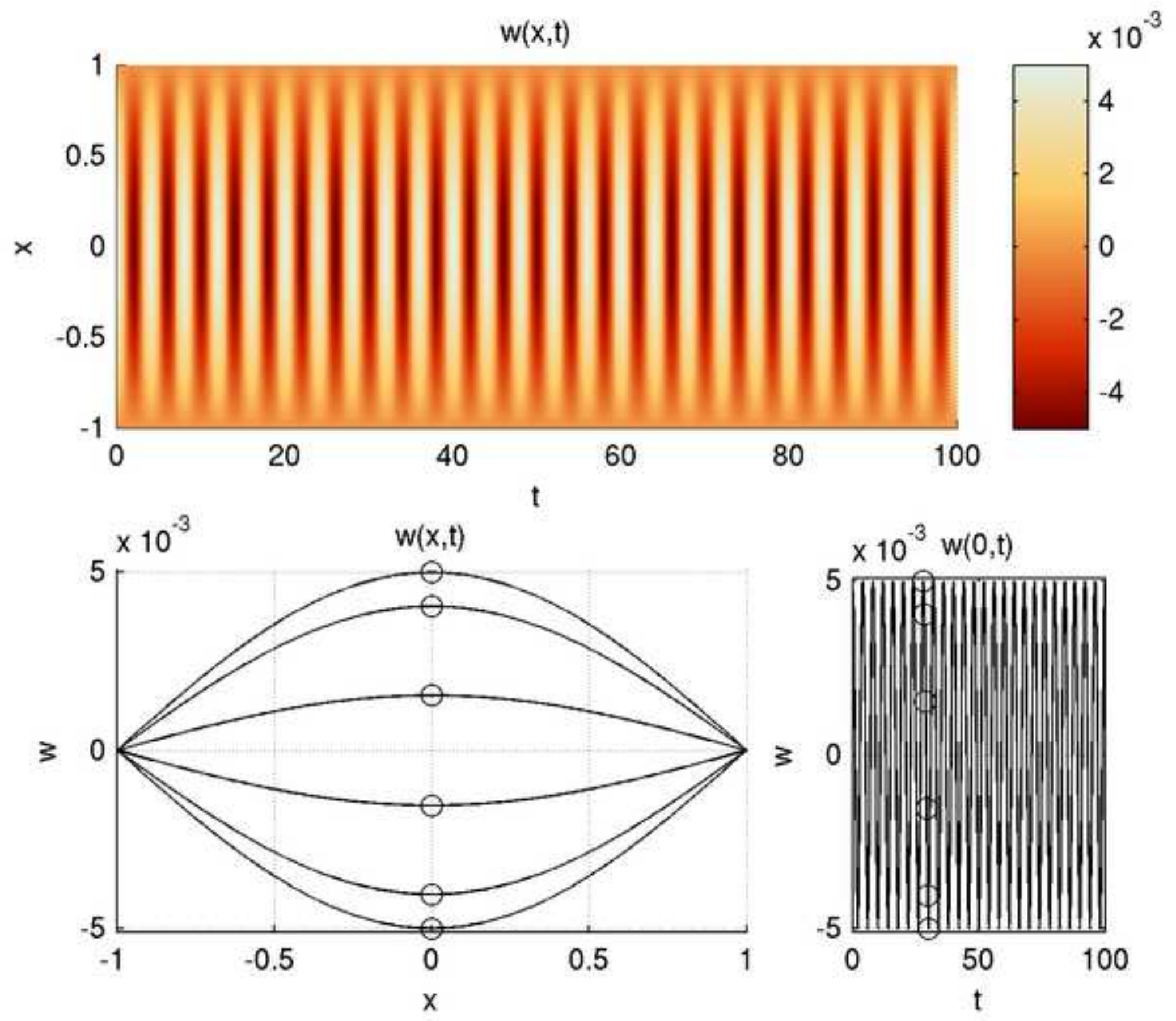

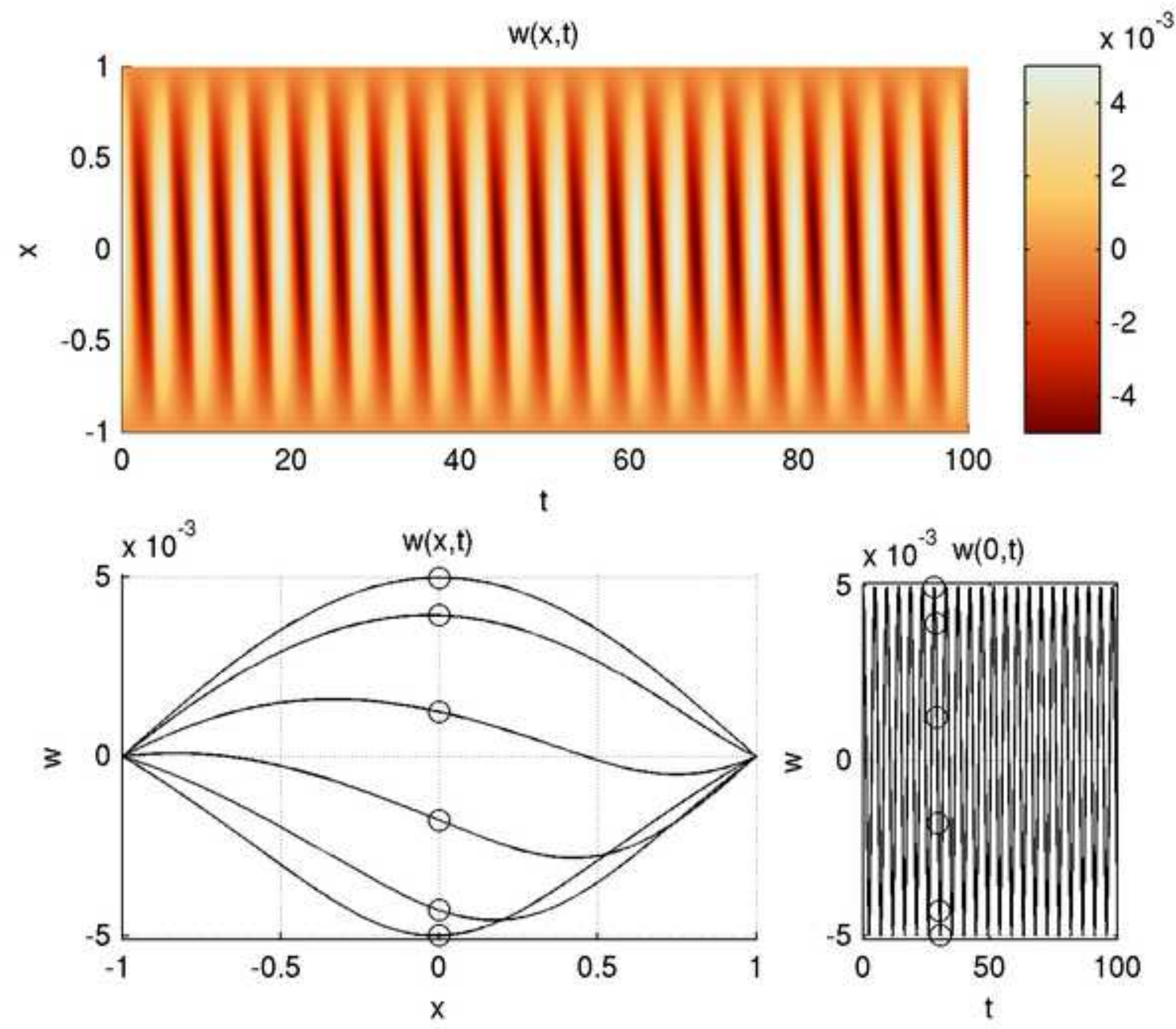

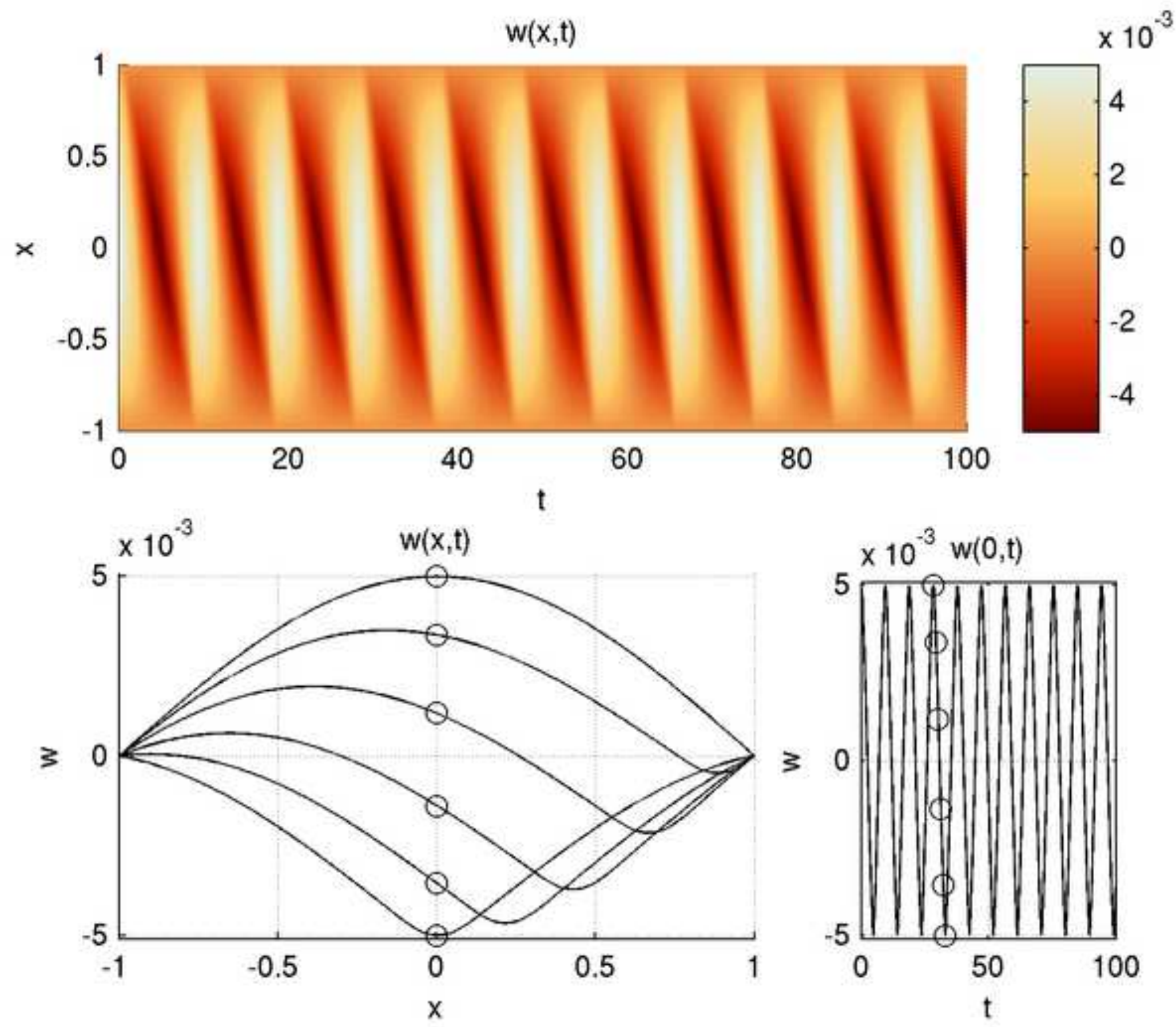

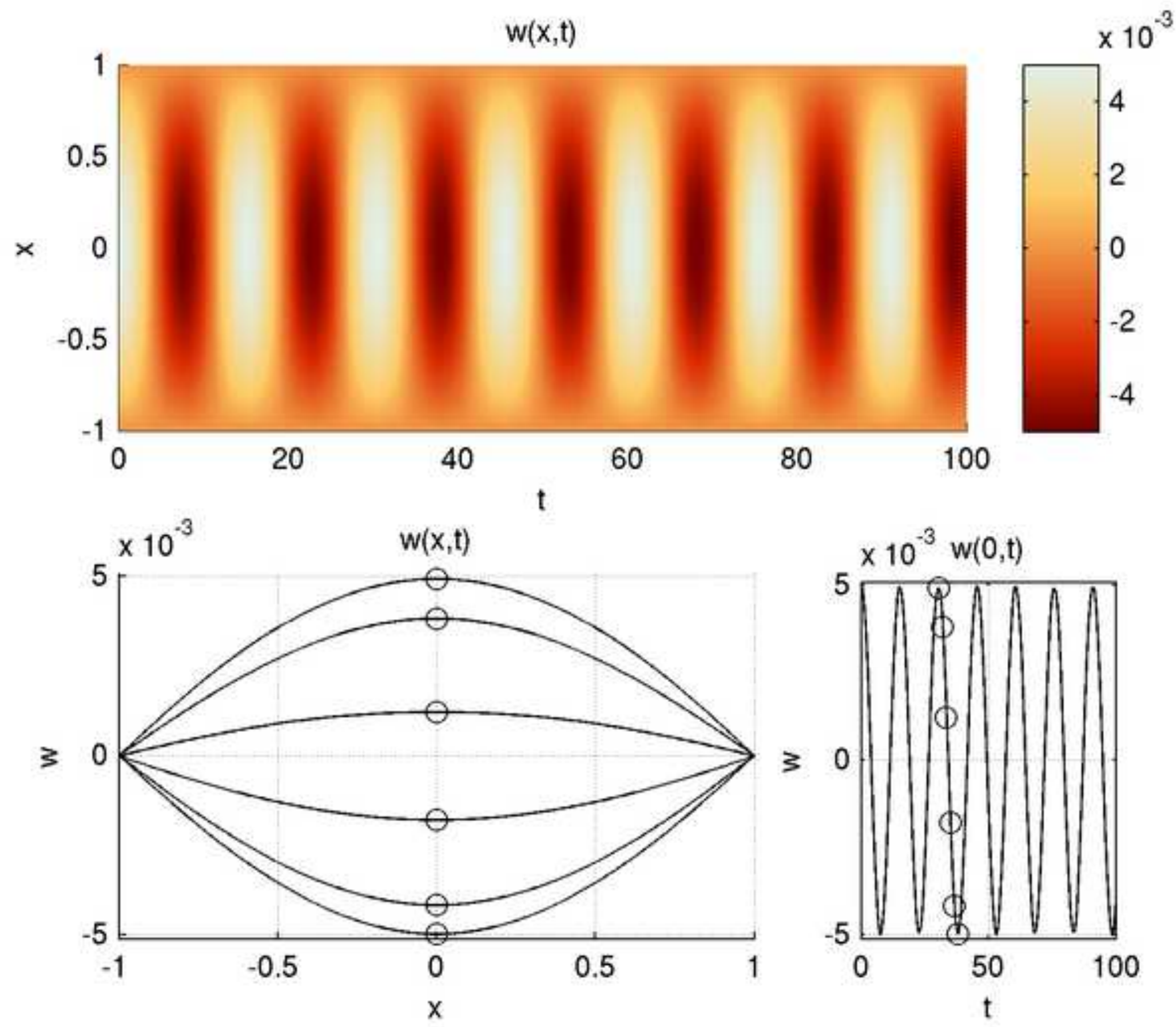

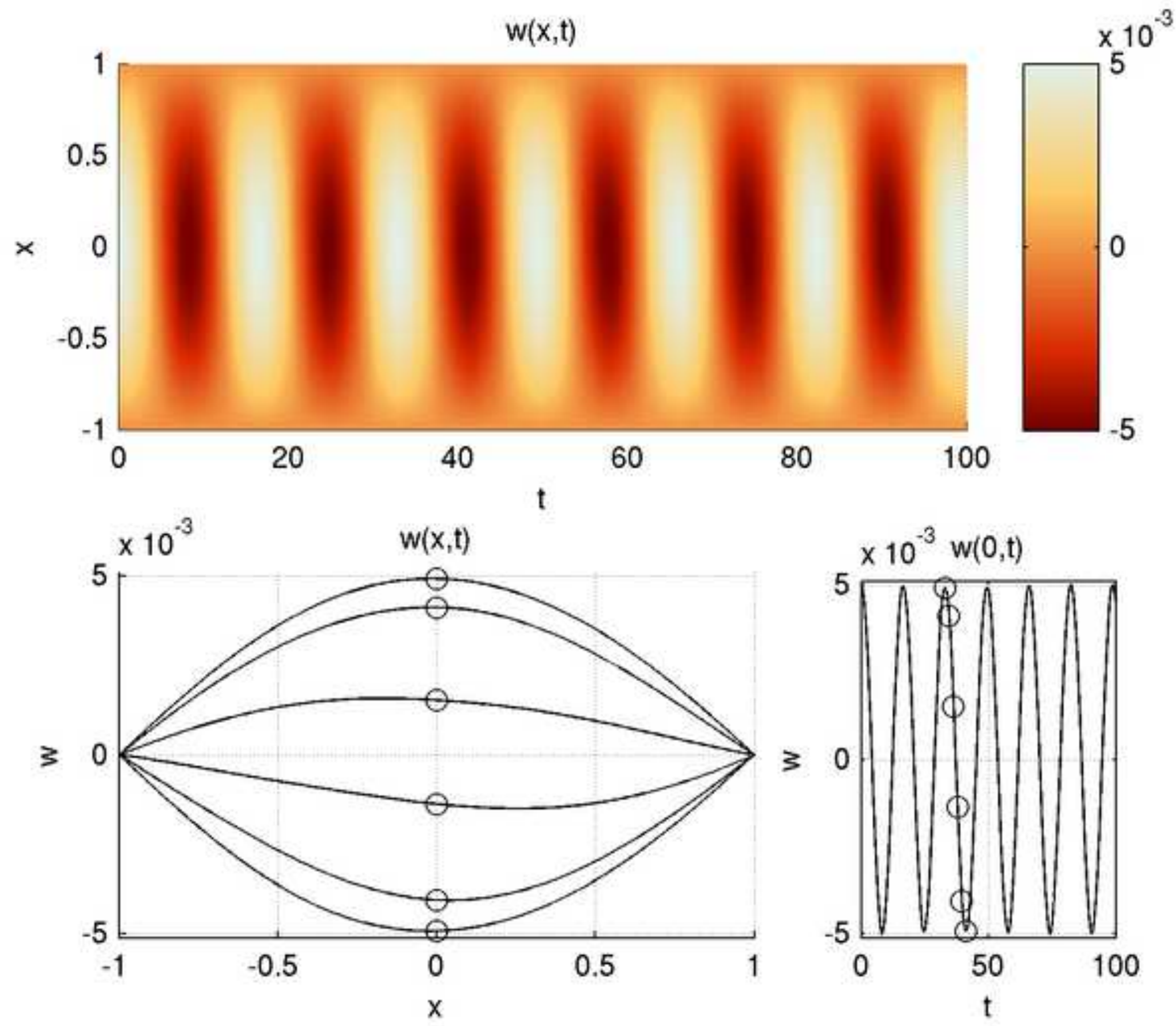

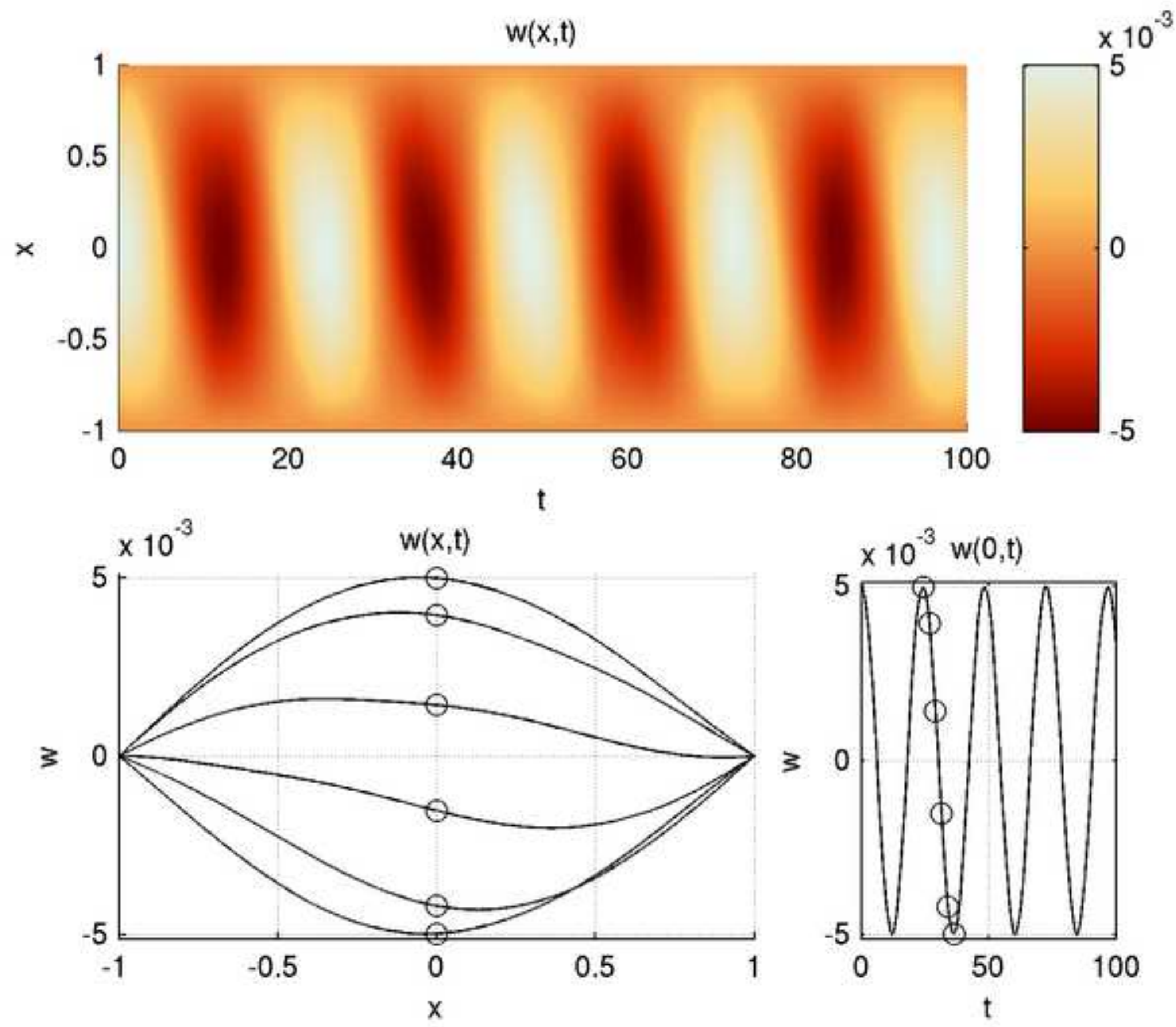

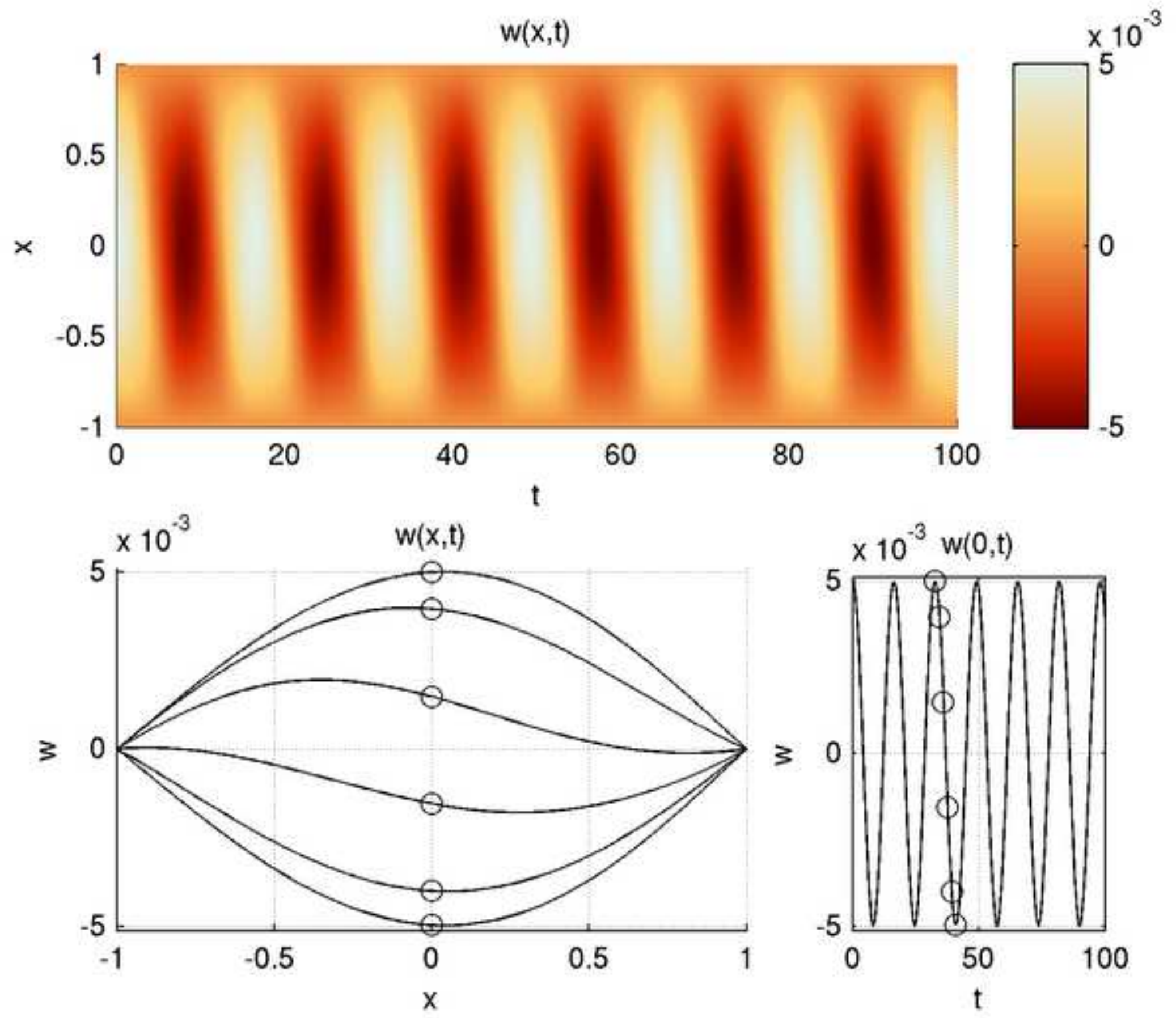

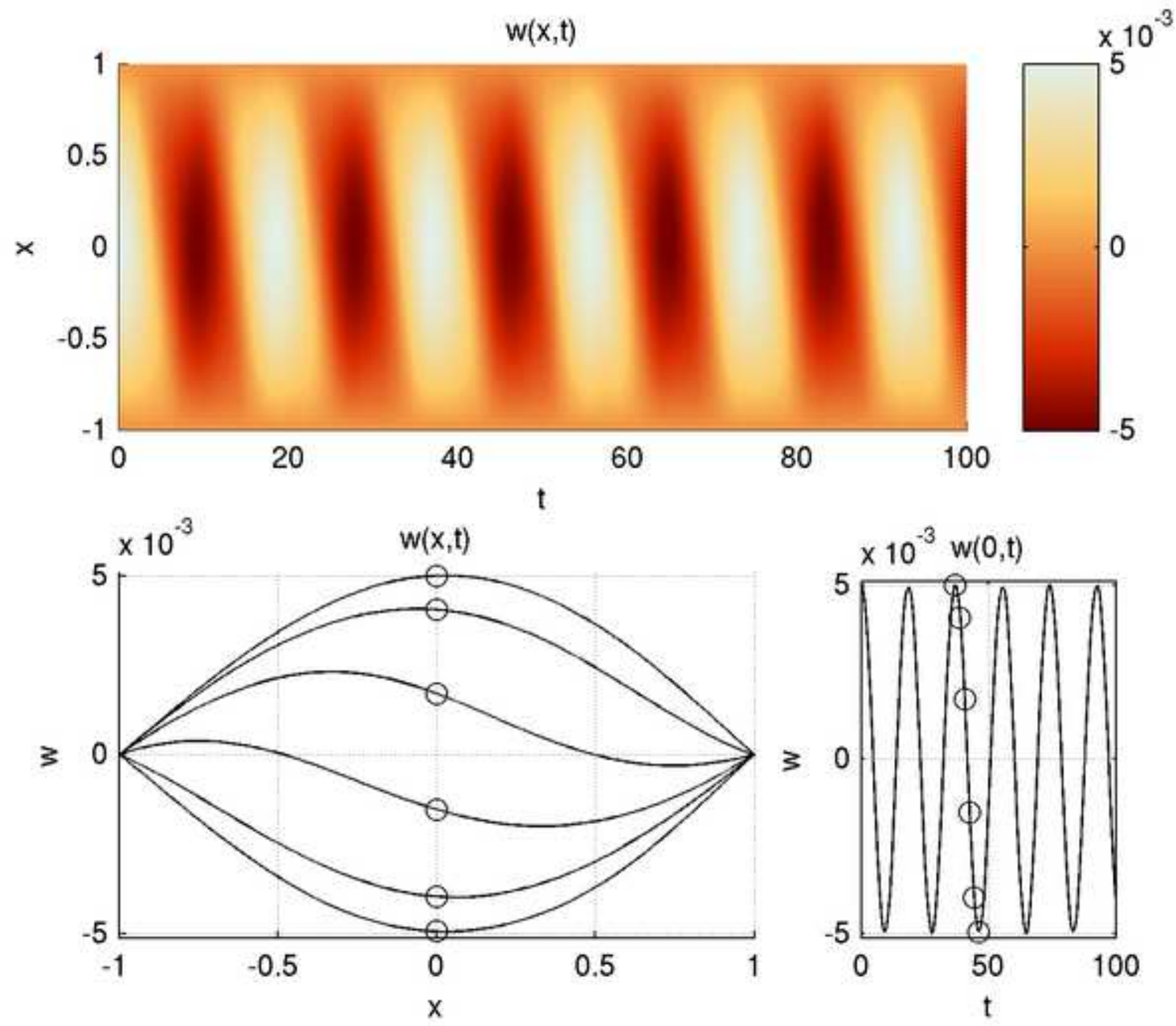

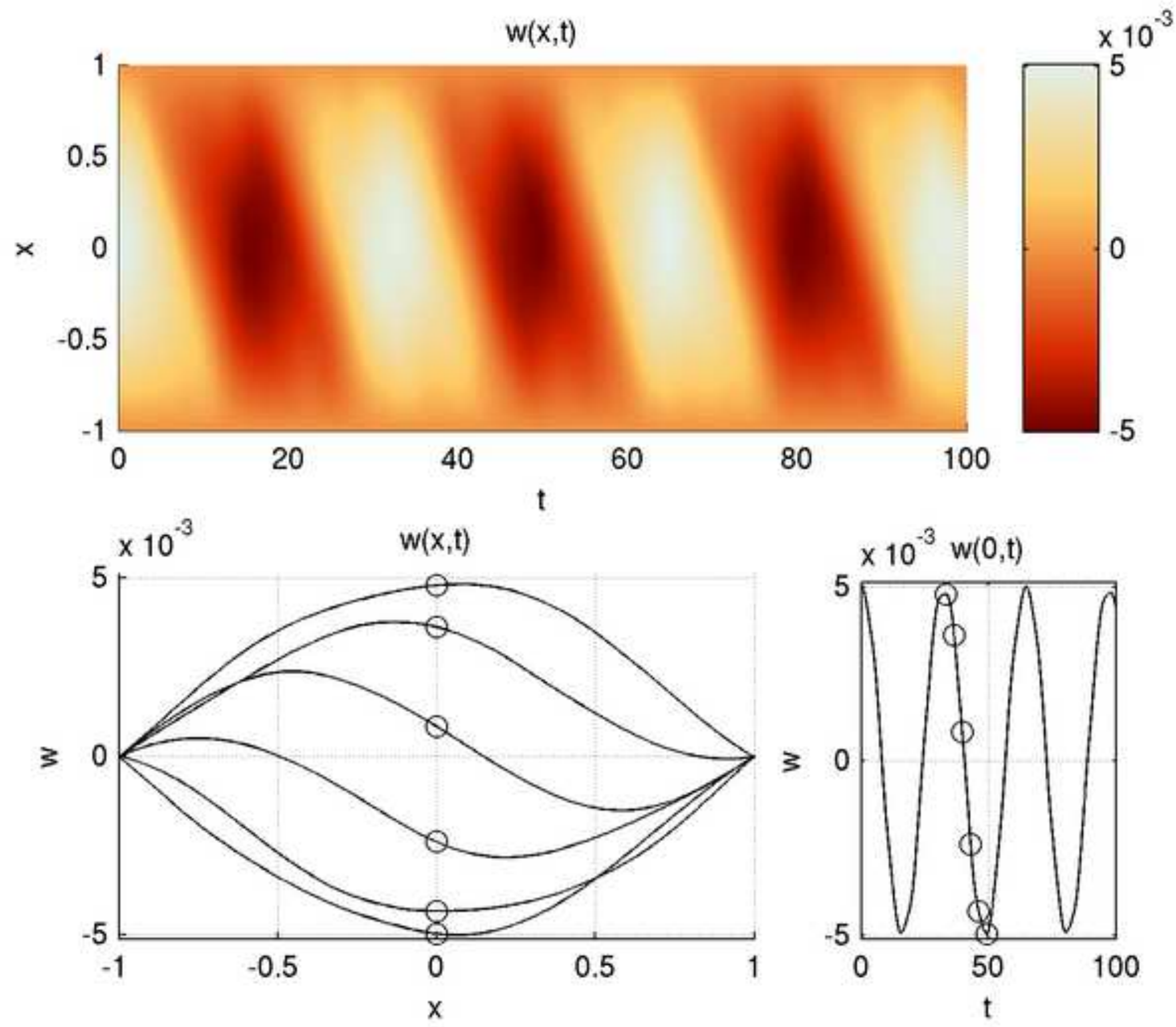

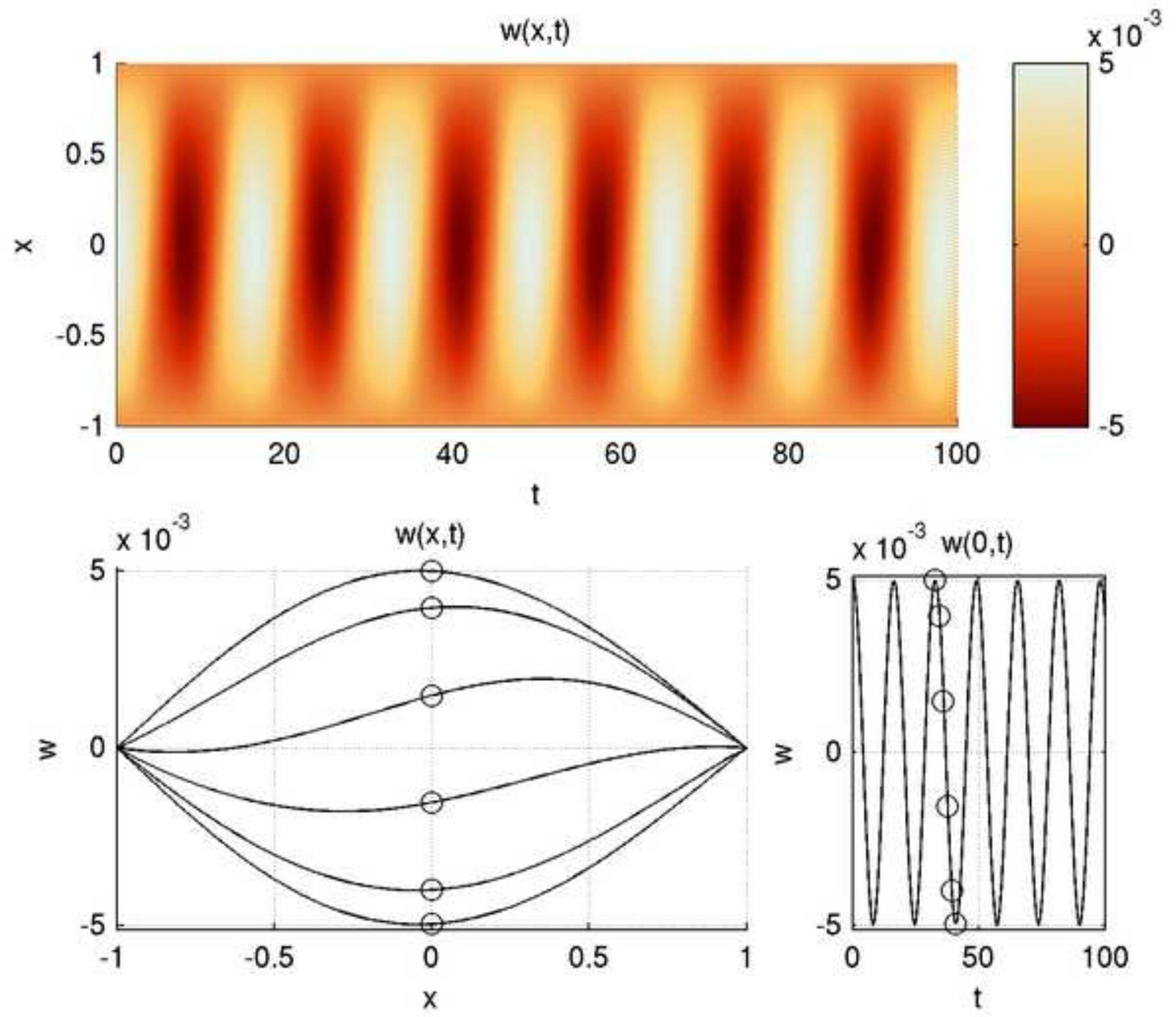

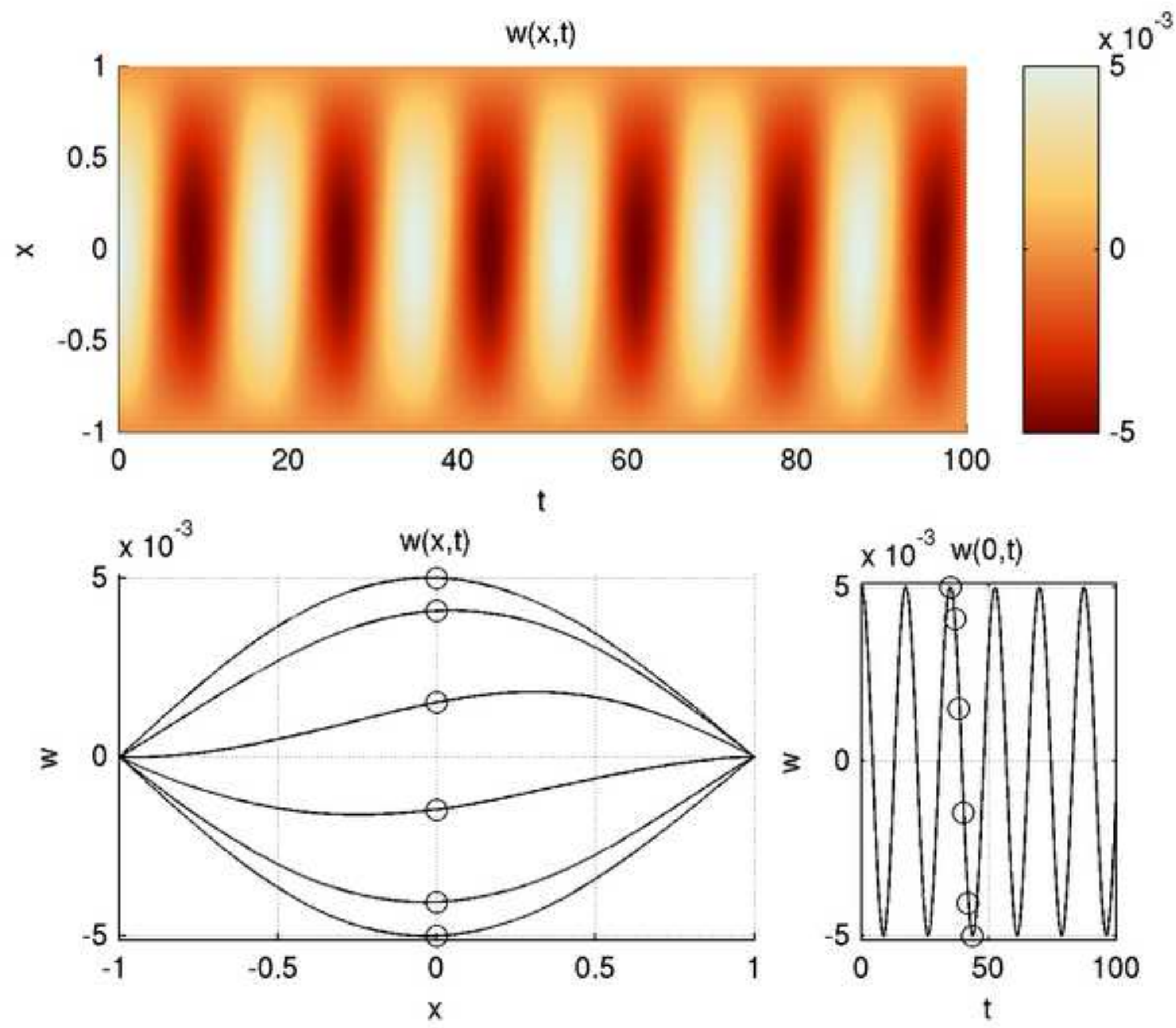

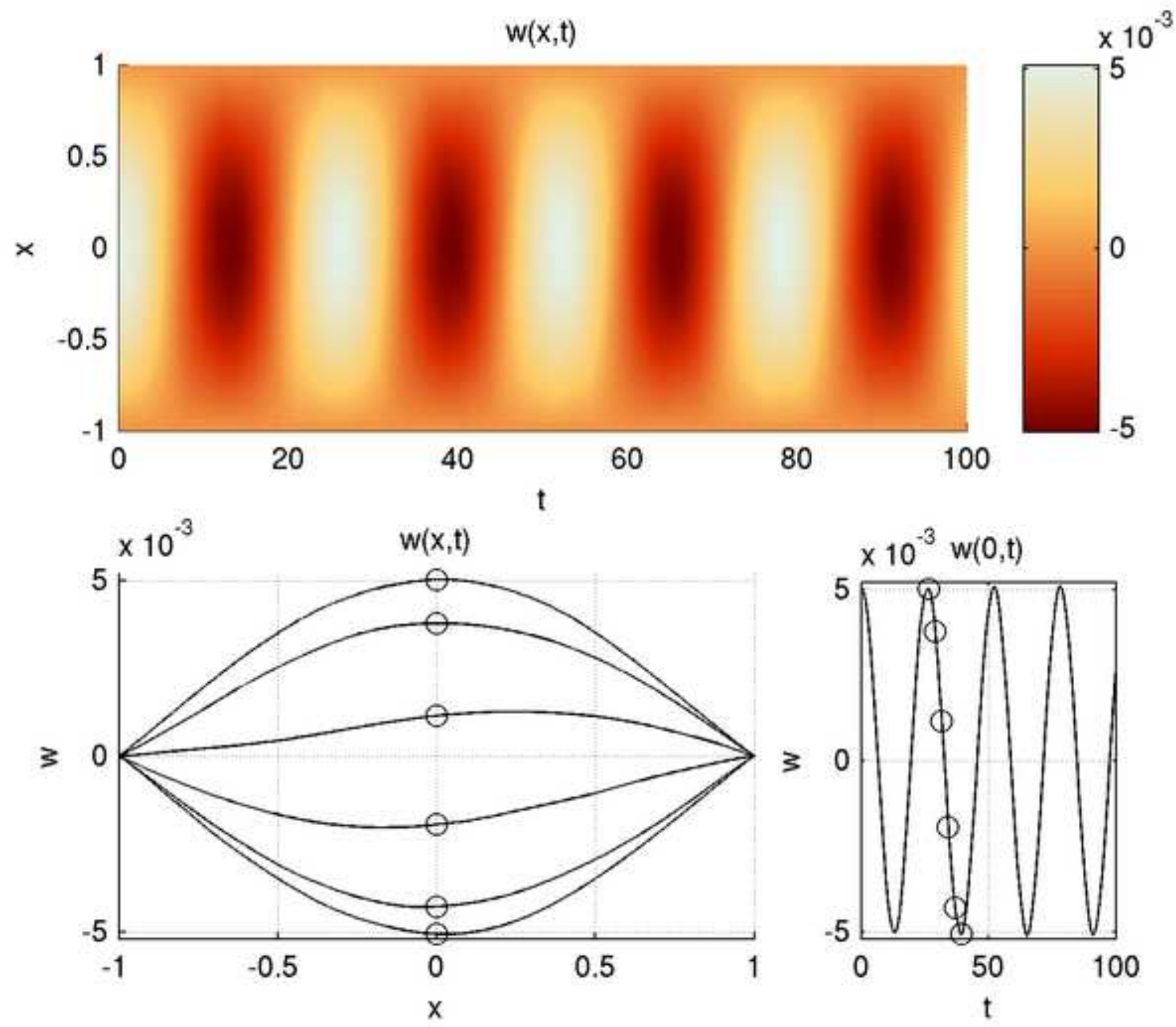
Nondimensional first natural frequency $\left(n_{0}=56\right)$

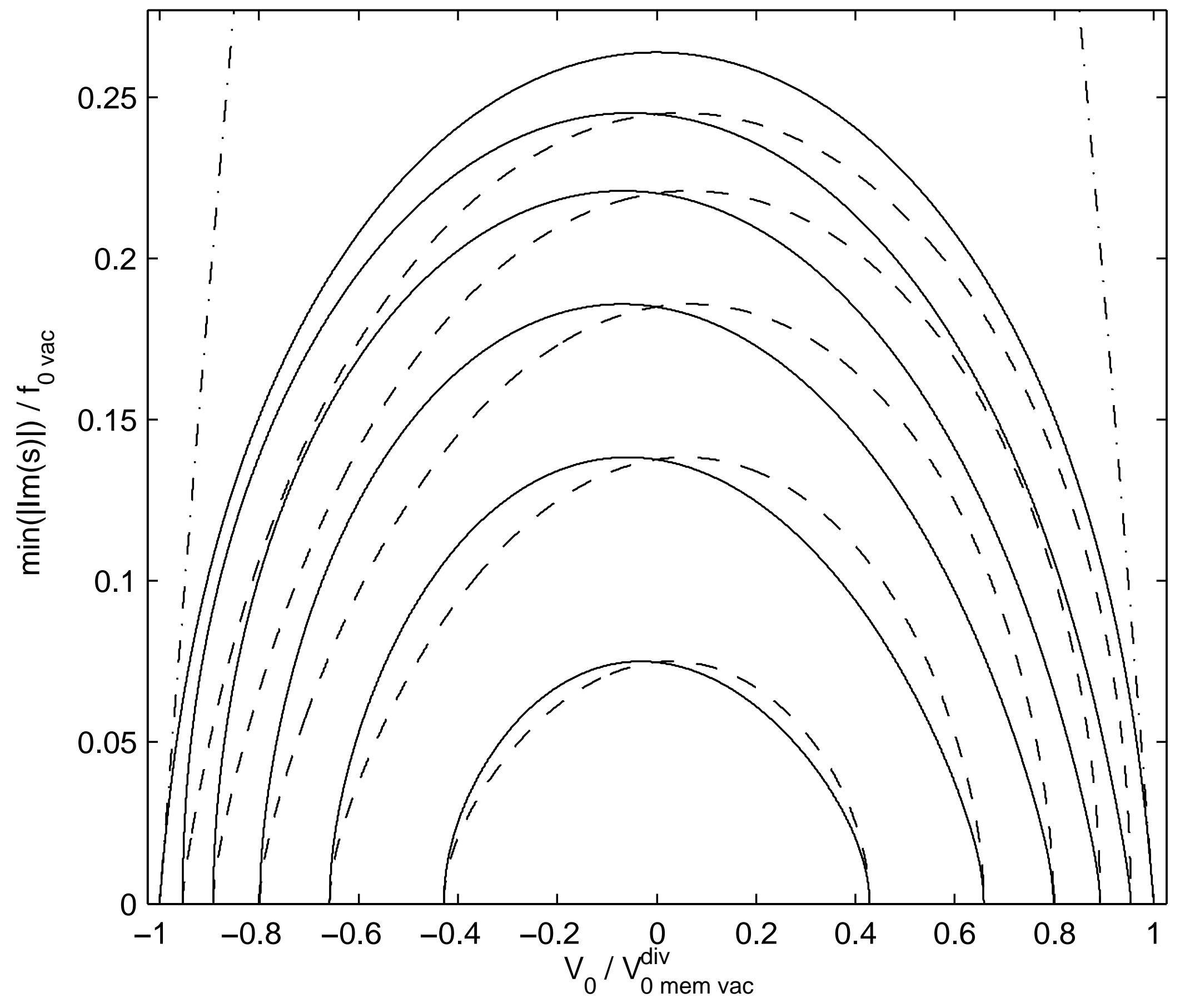



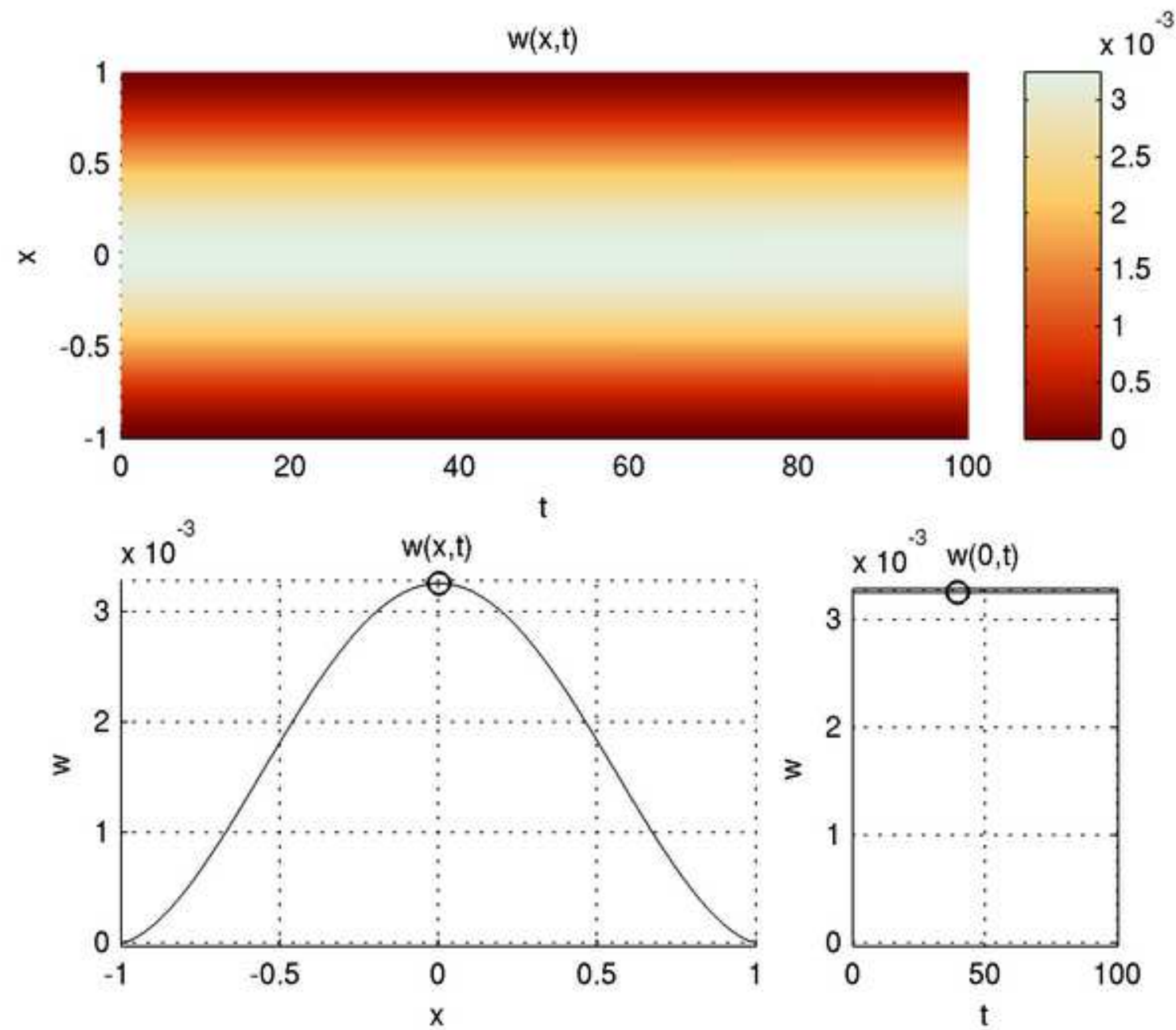